\title{
Ethnomedicinal, Phytochemical and Pharmacological Investigations of Perilla frutescens (L.) Britt.
}

\author{
Hiwa M. Ahmed $\mathbb{D}$
}

Sulaimani Polytechnic University, Slemani 46001, Kurdistan Regional Government, Iraq; hiwa2009@yahoo.com or hiwa.ahmed@spu.edu.iq

Received: 14 October 2018; Accepted: 2 December 2018; Published: 28 December 2018

\begin{abstract}
Perilla frutescens (L.) Britt. (PF) is an annual herbal medicinal, aromatic, functional food, and ornamental plant that belongs to the mint family, Lamiaceae. The origin of perilla traces back to East Asian countries (China, Japan, Korea, Taiwan, Vietnam, and India), where it has been used as a valuable source of culinary and traditional medicinal uses. The leaves, seeds, and stems of $P$. frutescens are used for various therapeutic applications in folk medicine. In the absence of a comprehensive review regarding all aspects of perilla, this review aims to present an overview pertaining to the botanical drug, ethnobotany, phytochemistry, and biological activity. It was found that the taxonomic classification of perilla species is quite confused, and the number of species is vague. Perilla has traditionally been prescribed to treat depression-related disease, anxiety, asthma, chest stuffiness, vomiting, coughs, colds, flus, phlegm, tumors, allergies, intoxication, fever, headache, stuffy nose, constipation, abdominal pain, and indigestion, and acts as an analgesic, anti-abortive agent, and a sedative. Until now, 271 natural molecules have been identified in perilla organs including phenolic acids, flavonoids, essential oils, triterpenes, carotenoids, phytosterols, fatty acids, tocopherols, and policosanols. In addition to solvent extracts, these individual compounds (rosmarinic acid, perillaldehyde, luteolin, apigenin, tormentic acid, and isoegomaketone) have attracted researchers' interest for its pharmacological properties. Perilla showed various biological activities such as antioxidant, antimicrobial, anti-allergic, antidepressant, anti-inflammatory, anticancer, and neuroprotection effects. Although the results are promising in preclinical studies (in vitro and in vivo), clinical studies are insufficient; therefore, further study needs to be done to validate its therapeutic effects and to ensure its safety and efficacy.
\end{abstract}

Keywords: bioactivity; essential oils; polyphenols; preclinical; rosmarinic acid; terpeniods

\section{Introduction}

Perilla frutescens (L.) Britt. is an annual herbal plant that belongs to the mint family, Lamiaceae [1,2]. It is commonly called perilla [3] or by other names (beefsteak plant, purple mint, perilla mint, Chines basil, Korean perilla, zisu in China, shiso in Japan, and tia to in Vietnam) [4]. It is widely cultivated throughout Asian countries such as China, Japan, South Korea, Vietnam, and India; however, China is probably considered to be a primary gene center for this species [1,2]. Perilla is historically an important herb that has been recorded in Chinese medical classics since around 500 A.D., especially in records named "Ming Yi Bie Lu" (Renown Physicians' Extra Records), and others where the herb is registered as a drug named "su" which means comforting the body and promoting the blood circulation. From the ancient times in the Song dynasty (960-1279 A.D.), it can be seen that the stem, leaf, and seed of the herb was equally commonly used. The drug items of the herb in traditional Chinese medicine are dried "Perilla leaf", "Perilla stalk" and "Perilla seed" corresponding to Folium Perillae, Caulis Perillae, and Fructus Perillae in the Chinese Pharmacopoeia (1990), while in the Japanese Pharmacopoeia (1991), Herba Perillae is listed as a drug derived from the leaves and twigs of perilla [5]. It has commonly 
been used as a traditional medicine and a functional food throughout Asian communities [6]. In the Chinese Pharmacopeia 2010, the dried parts of P. frutescens, such as stems Perillae Caulis (PCa), leaves Perillae Folium (PFo), and ripe fruits Perillae Fructus (PFr) are recorded for various therapeutic applications [4,7]. It has been used as a natural herbal medicine to recover from different symptoms, such as depression-related disease, asthma, anxiety, tumors, coughs, allergies, intoxication, cold, fever, chills, headache, stuffy nose, and some intestinal disorders [1,4,8-10]. It is used as an ornamental plant in gardens due to its wide morphological variability and attractive appearance [2]. It is also used as a kitchen herb in salads, sushi, soups, and as a spice, garnish, or food colorant as well. The seed oil is traditionally used to flavor foods [11]. Perilla also gains market importance in cosmetics, being processed in skin creams, soaps, and dermatological medicinal preparations, because of its biological activities [12]. The historical popularity and ethnopharmacological uses of this plant had attracted the interest of scientists to examine their pharmacological properties and resulted in a growing popularity among European countries as well. Interestingly, there are not many comprehensive scientific reviews to cover all aspects of information about perilla. Therefore, the purpose of this study is to provide an up-to-date summary in relation to the botanical, biological, phytochemical properties, and traditional uses in parallel with new perspectives of $P$. frutescens, and to provide an overview for future research on this plant.

\section{Methodology}

This review article was carried out to collect data from multiple databases, including PubMed, Web of Science, Wiley, Science Direct, Elsevier, American Chemical Society publications, SciFinder, and Google Scholar up to and including the year of 2018. The quality of the reviewed studies is well known, and only peer-reviewed articles were included, and considering only English literature that were available in the database; none of the studies in languages other than English were included for this review. No masters theses or Ph.D. dissertations, as well as unpublished articles, were included in this review. The pharmacological activities of perilla spices were reported only based on the extracted/isolated compounds directly found from perilla species, not commercially available unless otherwise stated, with appropriate controls in experiments. In silico study was not found in the literature and therefore excluded.

\section{Botanical Characteristics and Aspects of Cultivation}

The plant is a freely branching annual herb that grows up to $1.5 \mathrm{~m}$ high in some varieties. Stems are four-sided and covered with short hairs. Leaves are ovate, opposite, green to purple with toothed, crisped, laciniate, palmate, or serrate margins. They are glossy and downy-haired. The herb has a distinctive musky, mint-like odor. Flowers are small, bell-shaped, and a white or purple color with a distinctive ring of fine hairs along the bottom in terminal spikes or emerging from leaf axils, and four stamens are present in most species in that family with a gray-brown fruit $[2,13]$. The seeds are small and globular, and their 1000-seeds weight is about $4 \mathrm{~g}$ [3]. Its spreading is assured either by dropping close to the parent plant or they may be transported by wind or water. Perilla is said to resemble basil and coleus and may be confused with other members of the mint family.

According to Brenner [14] and Asif [3], the best diagnostic characteristics of perilla are the net-patterned testa and the distinctive smell of the crushed foliage. Perilla is reproduced via seeds. The cultivated varieties are generally grown using direct sowing or raised in nursery beds for transplanting in mid-spring. The optimal germination temperature is $20^{\circ} \mathrm{C}$, while it can be grown at slightly lower temperatures. The germination rate is fast and seed viability is lost relatively quickly over time; hence, in practice, fresh seeds are suggested to be used [2,13]. Perilla is a selfing [15], and a short-day plant in order to flower [16]. Harvesting is usually started at the end of September and the beginning of October [3], and this varies according to the intended use of the crop and climate condition in the area. 


\section{Classification and Taxonomy of Perilla}

The taxonomic classification and nomenclature of perilla seems to be controversial and different systems have been published [14]. Based on the decoration pattern and size of pollen grains, in China, cultivated taxa of perilla could be divided into five varieties: var. frutescens, var. arguta, var. crispa, var. auriculato-dentata, and var. acuta. From those varieties, the leaves of var. frutescens and var. acuta are usually used as fresh vegetables and to process pickles, var. crispa is used for its medicinal properties, and seeds of var. arguta are used for oil extraction because of its high seed yield [17]. It is believed that, based on morphological characters and uses, the genus of Perilla L. consists of only one species and two diverse cultivars, such as P. frutescens (L.) Britton var. frutescens as an oil seed crop and P. frutescens (L.) Britton var. crispa (Thunb.) W. Deane as a spicy vegetable and medicine, which are cross-fertile (in both varieties, phenotypes with green and purple shoots can be found) [2]. According to The Plant List (www.theplantlist.org), three varieties-var. frutescens, var. crispa (Thunb.) H.Deane, and var. hirtella (Nakai) Makino-are the accepted species. The anthocyanin-rich purple colored types are frequently used as food colorants in Japan and China [12].

\section{Ethnobotanical Uses}

Perilla has been one of the most popular species in the Eastern Asian communities, that has been used as an ingredient, for flavor and as a spice in cooking, garnish, soups, vegetable salad, sushi, as a food colorant, and to wrap and eat cooked food in Japan, India, and Korea $[4,18,19]$. In Korea, perilla seed oil is used for cooking and different industrial uses [18]. Moreover, seeds as spice also used to prepare sauce in India [19]. As an antidote, perilla leaf has been used in fish and crab dishes in China and Japan for a long time [9]. In India, the whole plant has been used to treat stomach disorders and for flavoring curries, and in combination with Artemisia scoparia is used as a refrigerant [20]. The seed is used for meat preservation and flavoring foods [21]. Its seed oil is used to massage twice a day for arthritis [22], used for earache in Nepal [23], as well as a condiment and in food preparation of rice cakes [24,25]. Leaves are used for cooking as vegetables [24]. In Vietnam, the leaves are used as a spice [26], while in China and Thailand, it is used in temperature regulation in the form of hot infusion (tea) [27]. Leaf juice is used to expel intestinal worms and cut wounds in Dekhatbhuli, Nepal [23]. Perilla root paste mixed with goat urine is used as a poultice for rheumatoid arthritis twice daily for one week [28].

In Japan, it has historically been used for making drying oil for waterproofing umbrellas or as lamp oil. Perilla is used as a culinary herb to color and flavor pickles, as well as a garnish for raw fish [29]. In Indochina, especially in towns, perilla leaves are used as a vegetable, while in mountainous areas and countryside, the leaves are not exploited but mericarps are eaten. The seeds of perilla (mericarps) are also used like sesame seeds. Perilla mericarps are roasted and mixed into steamed sticky rice often with cane sugar [29].

In human traditional herbal medicine in China and India, the stem of the plant is historically used as an analgesic and anti-abortion agent. The leaves are believed to be useful against asthma, colds, flu, chest stuffiness, vomiting, cough, constipation, and abdominal pain, as well as promoting stomach function $[19,30]$. According to the Chinese traditions, P. frutescens could be used to cure a number of conditions such as cold, fever, chills, headache, and stuffy nose [4]. In addition, the plant has traditionally been prescribed to treat depression-related disease, asthma, anxiety, tumors, coughs, allergies, intoxication, and some intestinal disorders [1,8-10].

The juice of the fresh leaves is utilized for curing injuries and the seed oil for massaging infants [19]. Other indications for using the leaves include dissipating colds, promoting the circulation of Qi [4], toning the stomach, and detoxification [4,9]. According to some further references, the stem of PF is used for promoting the circulation of Qi [4], pain relief, and preventing miscarriage [19,30]. The seed of perilla seems to exhibit useful properties too. Yu et al. [4] describe its activity in descending Qi and resolving phlegm, relieving cough and asthma, and loosening the bowel to relieve constipation. Perilla leaf is mentioned as an ingredient in many Chinese herbal preparations, such as "Ban Xia Hou Po" 
decoctions used against discomfort in the throat, and as an essential herbal remedy for psychological disorders such as generalized anxiety.

\section{Phytochemical Compounds in Perilla}

There are currently 271 various phytochemical compounds that have been isolated and reported in perilla seeds, stems, and leaves. Based on the chemical properties, these active compounds in perilla could be classified either as hydrophilic (phenolic acids, flavonoids, anthocyanins) or hydrophobic (lipophilic) ones (volatile compounds, triterpenes, phytosterols, fatty acids, tocopherols, and policosanols). The identified phytochemicals are listed in Tables 1-3, and some of their structures are displayed in Figures 1-4.

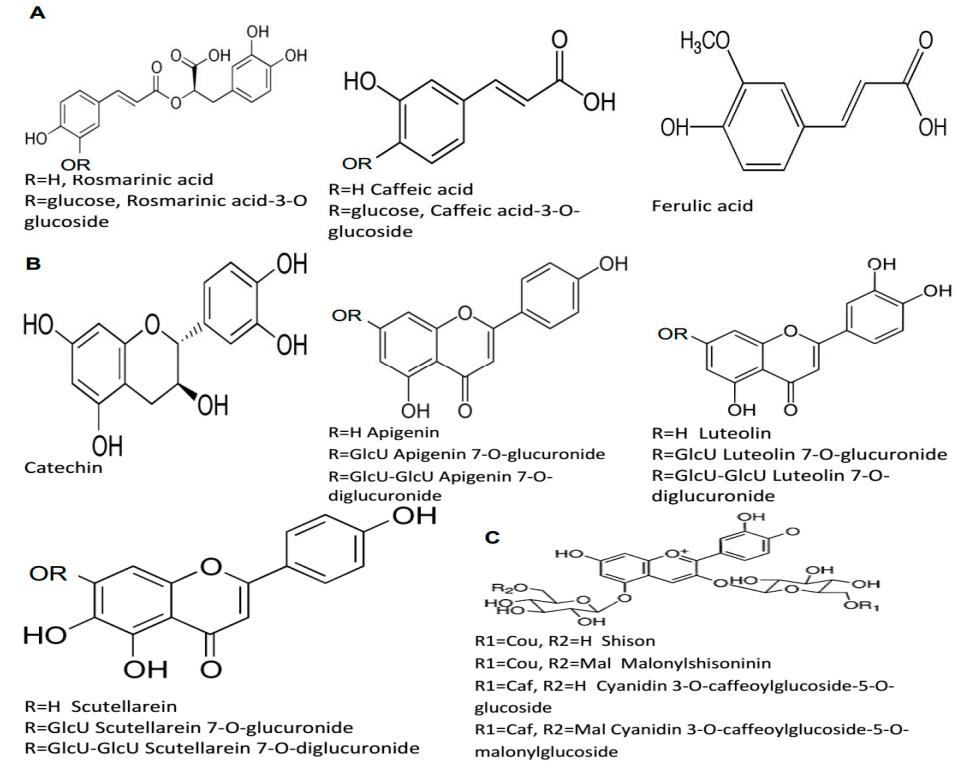

Figure 1. The chemical structures of chief hydrophilic compounds identified in P. frutescens: (A) phenolic acids; rosmarinic acid, rosmarinic acid-3-O-glucoside, caffeic acid, caffeic acid-3-O-glucoside, ferulic acid (B) flavonoids; catechin, apigenin, apigenin 7-O-glucuronide, apigenin 7-O-diglucuronide, luteolin, luteolin 7-O-glucuronide, luteolin 7-O-diglucuronide, scutellarein, scutellarein 7-O-glucuronide, scutellarein 7-O-diglucuronide, and (C) anthocyanins; shisonin, malonylshisonin, cyanidin 3-O-caffeoylglucoside-5-O-glucoside, cyanidin 3-O-caffeoylglucoside-5-O-malonylglucoside.

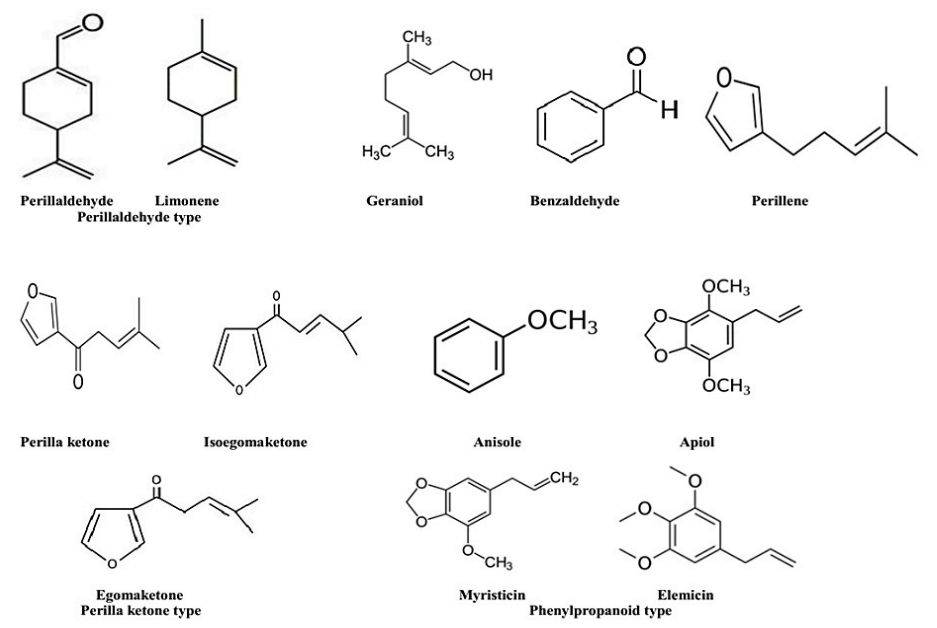

Figure 2. The chemical structures of some volatile compounds identified in P. frutescens; perillaldehyde, limonene, geraniol, perillene, benzaldehyde, perilla ketone, isoegomaketone, anisole, apiol, egomaketone, myristicin, elemicin. 


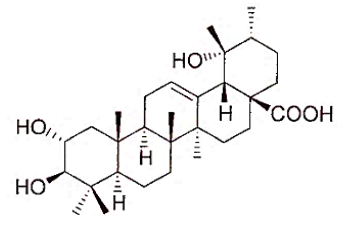

Tormentic acid

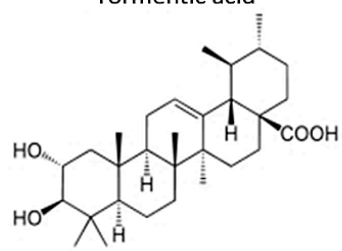

Corosolic acid

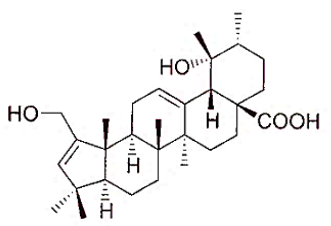

Hyptadienic acid

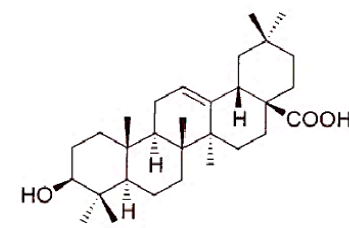

Oleanolic acid

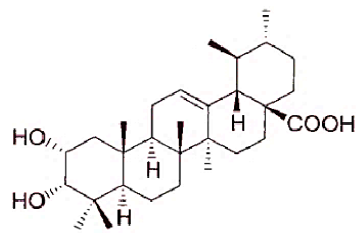

3-Epicorosolic acid

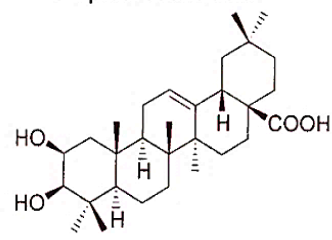

Augustic acid

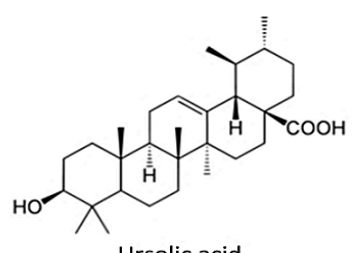

Ursolic acid

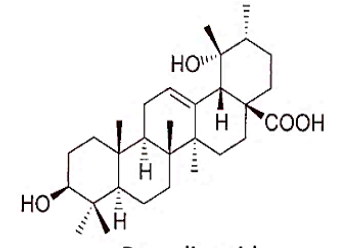

Pomolic acid

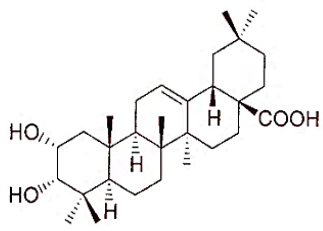

3-Epimaslinic acid

Figure 3. The chemical structures of triterpene acids identified in P. frutescens; tormentic acid, oleanolic acid, ursolic acid, corosolic acid, 3-Epicorosolic acid, pomolic acid, hyptadienic acid, augustic acid, 3-Epimaslinic acid.

A
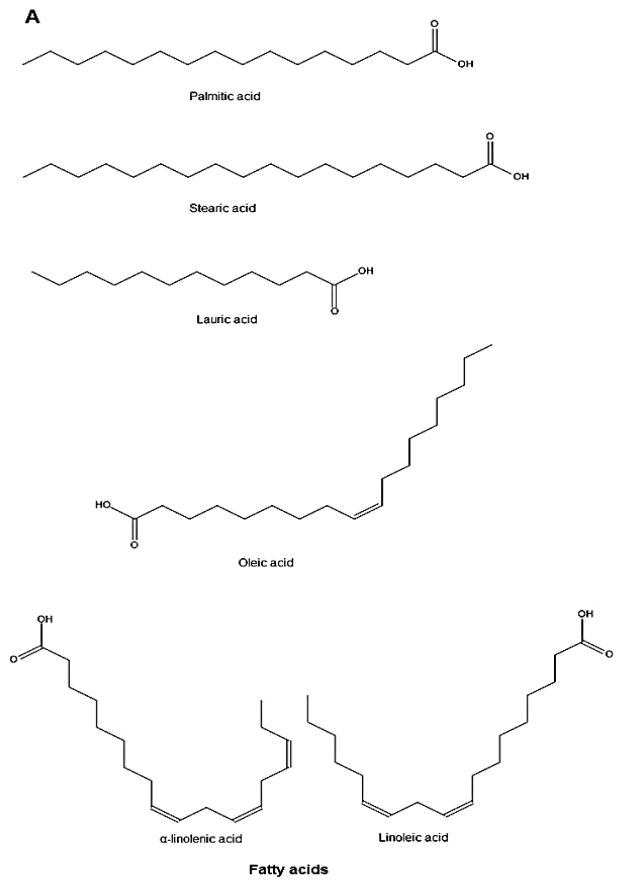

c

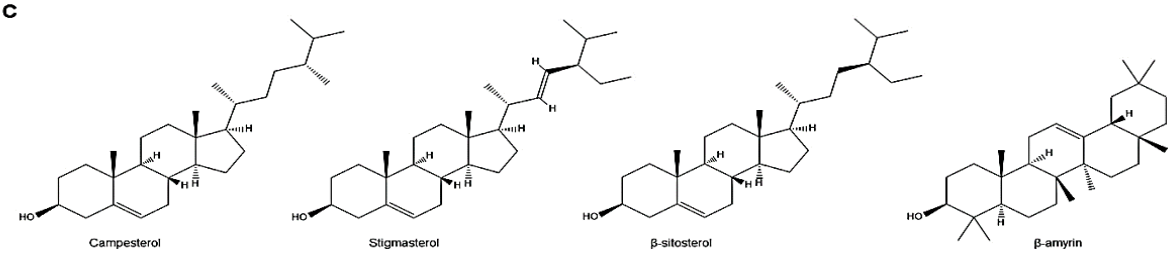

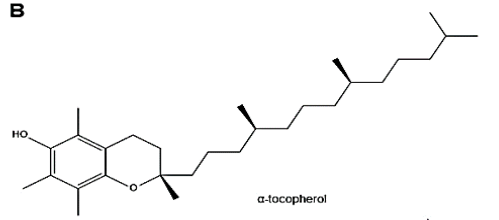
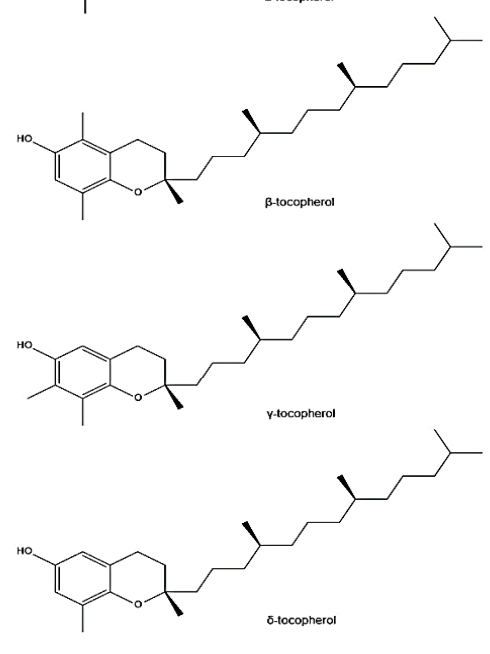

Phytosterols

Figure 4. The chemical structures of major hydrophobic compounds identified in P. frutescens: (A) fatty acids; palmitic acid, stearic acid, lauric acid, oleic acid, linoleic acid, linolenic acid (B) tocopherols; $\alpha$-tocopherol, $\beta$-tocopherol, $\gamma$-tocopherol, $\delta$-tocopherol and (C) phytosterols; campesterol, stigmasterol, $\beta$-sitosterol, $\beta$-amyrin. 


\subsection{Phenolic Compounds, Flavonoids, and Anthocyanins}

Phenolic compounds frequently occur in the perilla plant. They have a wide structural variability with a broad range of pharmacological activities (Table 1, Figure 1). Peng et al. [31] reported for the first time that catechin, ferulic acid, apigenin, luteolin, rosmarinic acid, and caffeic acid are determined by capillary electrophoresis in leaves and seeds of perilla. Similarly, Gu et al. [32] identified rosmarinic acid, luteolin, apigenin, and chrysoeriol by means of ultraviolet-visible spectroscopy, nuclear magnetic resonance spectroscopy (NMR) and electrospray ionisation mass spectrometry (ESI-MS) from the fruit of P. frutescens var. acuta. Meng et al. [11] identified various polyphenols from different varieties of perilla (var. crispa and var. frutescens) Britt., which included cinnamic acid derivatives (coumaroyl tartaric acid, caffeic acid, rosmarinic acid), flavonoids (apigenin 7-O-caffeoylglucoside, scutellarein 7-O-diglucuronide, luteolin 7-O-diglucuronide, apigenin 7-O-diglucuronide, luteolin 7-O-glucuronide, scutellarein 7-O-glucuronide), and anthocyanins (mainly cis-shisonin, shisonin, malonylshisonin and cyanidin 3-O-(E)-caffeoylglucoside-5-O-malonylglucoside). Zhou et al. [1] identified eleven phenolic compounds from cold-pressed $P$. frutescens var. arguta seed using column chromatography. These compounds were partly identical with the previously mentioned results: 30-dehydroxyl-rosmarinic acid-3-O-glucoside, rosmarinic acid-3-oglucoside, rosmarinic acid, rosmarinic acid methyl ester, luteolin, luteolin-5-O-glucoside, apigenin, caffeic acid, caffeic acid-3-O-glucoside, vanillic acid, and cimidahurinine using ion-trap time-of-flight mass spectrometry (IT-TOF MS) and nuclear magnetic resonance (NMR) analyses. Rosmarinic acid has been reported to be one of the chief phenolic compounds in perilla leaves [7] and recent study [33], showed more accumulation of phenolic components at the full flowering stage. It has been shown that the red color was given by the presence of a major anthocyanin, malonylshisonin, 3-O-(6-O-(E)-p-coumaryl- $\beta$-D-glucopyranosyl)-5-O-(6-O-malonyl- $\beta$-d-glucopyranosyl)-cyanidin [12], and other related anthocyanin compounds that accumulate in the epidermal cells of leaves and stems of the red-leaf chemotype [34]. The green-leaf chemotypes show only trace amounts of anthocyanin type compounds among all polyphenol compounds encountered [12].

Table 1. Phenolic acids, flavonoids, and anthocyanin compounds found in Perilla frutescens species.

\begin{tabular}{|c|c|c|c|}
\hline Number & Compounds & Plant Organs & References \\
\hline 1 & 30-dehydroxyl-rosmarinic acid-3-O-Glucoside & Seeds & [1] \\
\hline 3 & Caffeic acid-3-O-glucoside & Seeds & {$[1,8]$} \\
\hline 4 & Coumaroyl tartaric acid & Leaves & [11] \\
\hline 5 & Ferulic acid & Leaves, Seeds & {$[31]$} \\
\hline 7 & Rosmarinic acid methyl ester & Seeds & {$[1,35]$} \\
\hline 8 & Rosmarinic acid-3-O-glucoside & Seeds & {$[1,8]$} \\
\hline 9 & Vanillic acid & Seeds & [1] \\
\hline \multicolumn{4}{|c|}{ Flavonoids } \\
\hline 10 & $(+)$-catechin & Leaves, Seeds & [31] \\
\hline 15 & Catechin & Leaves, Seeds & [31] \\
\hline 16 & Cimidahurinine & Seeds & [1] \\
\hline 17 & Chrysoeriol & Seeds (fruits) & [32] \\
\hline 18 & Luteolin & Leaves, Seeds & {$[1,8,31,32]$} \\
\hline 19 & Luteolin 7-O-diglucuronide & Leaves & {$[11,34]$} \\
\hline 20 & Luteolin 7-O-glucoside & Leaves & [34] \\
\hline 21 & Luteolin 7-O-glucuronide & Leaves & [11] \\
\hline 22 & Luteolin-5-O-glucoside & Seeds & [1] \\
\hline 23 & Scutellarein & Leaves & {$[11,34]$} \\
\hline 24 & Scutellarein 7-O-diglucuronide & Leaves & {$[11,34]$} \\
\hline
\end{tabular}


Table 1. Cont.

\begin{tabular}{|c|c|c|c|}
\hline Number & Compounds & Plant Organs & References \\
\hline \multicolumn{4}{|c|}{ Anthocyanins } \\
\hline 25 & Chrysontenin & Leaves & [35] \\
\hline 27 & cis-Shisonin & Leaves & [11] \\
\hline 28 & Cyanidin 3-O-caffeoylglucoside-5-O-glucoside & Leaves, Stems & [34] \\
\hline 29 & Cyanidin 3-O-[E]-caffeoylglucoside-5-O-malonylglucoside & Leaves & [11] \\
\hline 32 & Cyanidin-3-O-(6-Ocoumaroyl)glucoside & Leaves & [35] \\
\hline 33 & Cyanidin-3-O-(6-Ocoumaroyl)glucoside-5-O-glucoside & Leaves & [35] \\
\hline 34 & Cyanin & Leaves, Stems & {$[34,35]$} \\
\hline 35 & Malonylshisonin & Leaves, Stems & {$[11,34]$} \\
\hline 36 & Peonidin 3-O-malonylglucoside-5-O-p-coumarolglucoside & Leaves, Stems & [34] \\
\hline
\end{tabular}

\subsection{Volatile Compounds}

Essential oils (EOs) are a type of secondary metabolites that can be extracted from different aromatic plant organs such as flowers, buds, stems, bark, leaves, fruits, etc. [36]. Their most frequent components are terpenoids, and aromatic and aliphatic compounds. A number of varieties are distinguished by the various chemical compositions of the essential oils extracted from their plant organs as a primary component of the oil known as chemotypes. According to Ito, Zhang, and Ghimire et al. [37-39], based on the main components of the EOs, different chemotypes have been described in perilla such as perillaketone (PK) (isoegomaketone), perillaldehyde (PA), elsholtziaketone (EK), citral (C), phenylpropanoids (PP) (myristicin, dillapiole, elemicin), perillene (PL), beta-caryophyllene, myristicine (MT), limonene, and piperitenone (PT). EOs are known to possess various bioactivities including antibacterial, antiviral, antifungal, anti-inflammatory, antimutagenic, anticarcinogenic, antidiabetic, antiprotozoal, and antioxidant [36].

The composition of EO of different organs of the perilla plant has been frequently analyzed and, until present, 193 different compounds have been identified (Table 2, Figure 2). The method of extraction may have a significant effect on the composition of the extracts too. Huang et al. [40] compared hydrodistillation, supercritical fluid extraction (SFE-CO2), and headspace solid phase microextraction (HS-SPME), followed by gas chromatography-mass spectrometry (GC-MS) analysis of volatile compounds and they found 64 compounds, mainly perillaldehyde and perilla ketone. Tian et al. [41] identified 119 compounds from the essential oil of perilla from eleven different areas, of which the predominant compounds were 2-acetylfuran (max. 82.17\%), perillaldehyde (max. $53.41 \%$ ), caryophyllene (max. 38.34\%), laurolene (max. 40.6\%), 2-hexanoylfuran (max. 33.03\%), 2-butylamine (max. 22.22\%), $\alpha$-asarone (max. 11.85\%), farnesene (max. 9.25\%), $\alpha$-caryophyllene (max. 9.16\%), and $(\mathrm{Z}, \mathrm{E})$-farnesene (max. $7.14 \%$ ). Sixty-five aromatic compounds were identified from ten perilla accessions with the predominance of perillaldehyde, perilla ketone, $\beta$-dehydro-elsholtzia ketone, limonene, shisofuran, farnesene $(\mathrm{Z}, \mathrm{E}, \alpha), \beta$-caryophyllene, and trans-shisool [42]. 
Table 2. Volatile oil compounds found in Perilla frutescens species.

\begin{tabular}{|c|c|c|c|}
\hline Number & Compounds & Parts & References \\
\hline 40 & $(\mathrm{E}, \mathrm{E})-\alpha$-Farnesene & Leaves & [40] \\
\hline 41 & (Z)-3-Hexenyl acetate & Leaves & {$[40,43]$} \\
\hline 42 & $(\mathrm{Z}, \mathrm{E})-\alpha$-Farnesene & Leaves & {$[40,41,44]$} \\
\hline 43 & 1-(3-Cyclohexen-1-yl)-2,2-dimethyl-1-propanone & Leaves & [40] \\
\hline 44 & 1,10-Decanediol & Leaves & [41] \\
\hline 45 & 1,2-Benzenedicarboxylic acid & Leaves & [7] \\
\hline 46 & 1,4,7,-Cycloundecatriene, 1,5,9,9-Tetramethyl-, Z,Z,Z- & Stems, Leaves, Seeds & [7] \\
\hline 47 & 1,6-Cyclodecadiene & Leaves & [7] \\
\hline 48 & 10-Undecyn-1-ol & Leaves & [41] \\
\hline 49 & 1-Cyclohexane-1-carboxaldehyde & Stems, Leaves, Seeds & {$[7]$} \\
\hline 50 & 1-Cyclohexene-1-methanol & Leaves & [41] \\
\hline 51 & 1-Octen-3-ol & Leaves & {$[40,41,43,44]$} \\
\hline 52 & 2,2-Dimethylpentane & Leaves & [41] \\
\hline 53 & 2,4,6-Triisopropylphenol & Leaves & [40] \\
\hline 54 & 2,4-Hexadienal & Leaves & {$[40,41]$} \\
\hline 55 & 2-Acetyl-5-methyl furan & Leaves & [43] \\
\hline 56 & 2-Acetylfuran & Leaves & [41] \\
\hline 57 & 2-Butylamine & Leaves & [41] \\
\hline 58 & 2-Cyclopentenone & Leaves & [41] \\
\hline 59 & 2-Ethyladamantane & Leaves & [40] \\
\hline 60 & 2-Hexanoylfuran & Stems, Leaves, Seeds & {$[7,41]$} \\
\hline 61 & 2-Hexenal & Leaves & {$[40,41]$} \\
\hline 62 & 2-Hexenal & Leaves & [43] \\
\hline 63 & 2-Hydroxypyridine & Leaves & [41] \\
\hline 64 & 2-Isopropylidene-3-methyl-hexa-3,5-dienal & Leaves & [40] \\
\hline 65 & 2-Methoxy-3-propenyl-phenol & Leaves & [7] \\
\hline 66 & 2-Methyl-2-cyclopentenone & Leaves & [41] \\
\hline 67 & 2-Methylcyclopentanone & Leaves & [41] \\
\hline 68 & 2-Nonyne & Leaves & [41] \\
\hline 69 & 3,5-Diethyl-toluene & Leaves & [40] \\
\hline 70 & 3-Octanol & Leaves & [45] \\
\hline 71 & 4,4-Dimethyl-2-cyclopenten-1-one & Leaves & [41] \\
\hline 72 & 4-Tert-pentylphenol & Leaves & [40] \\
\hline 73 & Acetophenone & Leaves & [40] \\
\hline 74 & Acetyl eugenol & Leaves & {$[40]$} \\
\hline 75 & a-Cubebene & Leaves & [40] \\
\hline 76 & Alloaromadendrene & Leaves & [41] \\
\hline 77 & All-trans-squalene & Leaves & [40] \\
\hline 78 & Anisole & Stems & [7] \\
\hline 79 & Apiol & Leaves & {$[40,41]$} \\
\hline 80 & Asarone & Leaves & {$[7,41]$} \\
\hline 81 & $a$-Terpinyl acetate & Leaves & [43] \\
\hline 82 & Benzaldehyde & Leaves & {$[40,41,44]$} \\
\hline 83 & Benzene acetaldehyde & Leaves & [40] \\
\hline 84 & Bornyl acetate & Leaves & {$[40,45]$} \\
\hline 85 & Cadina-3,9-diene & Leaves & [40] \\
\hline 86 & Calarene & Leaves & [41] \\
\hline 87 & Camphane & Leaves & [41] \\
\hline 88 & Camphene & Leaves & {$[40,41,43]$} \\
\hline 89 & Carvone & Leaves & [45] \\
\hline 90 & Caryophyllene & Stems, Leaves & {$[7,40,41,45]$} \\
\hline 91 & Caryophyllene oxide & Leaves, Seeds & {$[7,40,41,43-45]$} \\
\hline 92 & cis-Pyranoid & Leaves & [45] \\
\hline 93 & cis-(Z)- $\alpha$-Bisabolene epoxide & Leaves & [41] \\
\hline 94 & cis-Asarone & Leaves & [41] \\
\hline 95 & cis-Geranio & Leaves & [40] \\
\hline 96 & cis-Lanceol & Leaves & [40] \\
\hline 97 & cis-Nerolidol & Leaves & [41] \\
\hline 98 & cis-Ocimene & Leaves & [41] \\
\hline 99 & cis-Verbenol & Leaves & [41] \\
\hline 100 & Cosmene & Leaves & [41] \\
\hline 101 & Cuminaldehyde & Leaves & [43] \\
\hline 102 & Curlone & Stems & [7] \\
\hline 103 & Cycloheptane & Leaves & [41] \\
\hline 104 & Cyclohexanone & Leaves & [41] \\
\hline 105 & Decane & Leaves & [41] \\
\hline
\end{tabular}


Table 2. Cont.

\begin{tabular}{|c|c|c|c|}
\hline Number & Compounds & Parts & References \\
\hline 106 & Dihydrocarveol & Leaves & {$[41,45]$} \\
\hline 107 & Dihydrocarveol acetate & Leaves & {$[41,45]$} \\
\hline 108 & Dodecane & Leaves & [41] \\
\hline 109 & Egomaketone & Leaves & {$[45,46]$} \\
\hline 110 & Elemicin & Leaves & {$[29,41,45]$} \\
\hline 111 & Elixene & Leaves & [7] \\
\hline 112 & Elsholtziaketone & Leaves & [44] \\
\hline 113 & $\varepsilon$-Muurolene & Leaves & [41] \\
\hline 114 & Eremophilene & Leaves & [41] \\
\hline 115 & Eucalyptol & Leaves & {$[41,45]$} \\
\hline 116 & Farnesol & Leaves & {$[41,43]$} \\
\hline 117 & Furfuryl alcohol & Leaves & {$[41]$} \\
\hline 118 & Geraniol & Leaves & [41] \\
\hline 119 & Germacrene D & Leaves & {$[40,41,44]$} \\
\hline 120 & Germacrene D-4-ol & Leaves & [40] \\
\hline 121 & Heneicosane & Leaves & [41] \\
\hline 122 & Hexadecane & Leaves & [41] \\
\hline 123 & Hexahydro farnesyl acetone & Leaves & [44] \\
\hline 124 & Humulene epoxide II & Leaves & {$[41,45]$} \\
\hline 125 & Humulene epoxide-II & Leaves & {$[44]$} \\
\hline 126 & Isobornyl acetate & Leaves & [40] \\
\hline 127 & Isocaryophyllene & Leaves & [41] \\
\hline 128 & Isoegomaketone & Leaves & [44] \\
\hline 129 & Isoelemicin & Leaves & [41] \\
\hline 130 & Isoeugenol & Leaves & [41] \\
\hline 131 & Isolimonene & Leaves & [41] \\
\hline 132 & Isomenthone & Leaves & [41] \\
\hline 133 & Isopulegone & Leaves & [41] \\
\hline 134 & Laurolene & Leaves & [41] \\
\hline 135 & Limonen oxide, cis & Leaves & {$[45]$} \\
\hline 136 & Limonene & Leaves & {$[29,40,41,43,45]$} \\
\hline 137 & Limonene oxide & Leaves & [45] \\
\hline 138 & Limonene oxide, trans & Leaves & [45] \\
\hline 139 & Linalool oxide & Leaves & [45] \\
\hline 140 & Linalool oxide trans & Leaves & [45] \\
\hline 141 & Linalyl oxide $c i s$ & Leaves & [45] \\
\hline 142 & Longifolene & Leaves & [41] \\
\hline 143 & Longipinocarvone & Leaves & [40] \\
\hline 144 & Massoia lactone & Leaves & {$[41]$} \\
\hline 145 & Menthol & Leaves & {$[41,47]$} \\
\hline 146 & Menthone & Leaves & [41] \\
\hline 147 & Methyl chavicol & Leaves & [43] \\
\hline 148 & Methyl eugenol & Leaves & [41] \\
\hline 149 & Methyl geranate & Leaves & {$[40,41,45]$} \\
\hline 150 & Methyl heptenone & Leaves & [41] \\
\hline 151 & Methyl isoeugenol & Leaves & [41] \\
\hline 152 & Methyl thymyl ether & Seeds & [7] \\
\hline 153 & M-Mentha-6,8-diene & Leaves & {$[40]$} \\
\hline 154 & Myrcene & Leaves & {$[41,45]$} \\
\hline 155 & Myristicin & Leaves & {$[29,40,41,45]$} \\
\hline 156 & Naginata ketone & Leaves & [41] \\
\hline 157 & Nerol acetate & Leaves & [41] \\
\hline 158 & $n$-Heptadecane & Leaves & [41] \\
\hline 159 & Nonacosane & Leaves & [40] \\
\hline 160 & Nonane & Leaves & [41] \\
\hline 161 & $n$-Tricosane & Leaves & [41] \\
\hline 162 & Octacosane & Leaves & {$[40]$} \\
\hline 163 & Patchoulane & Leaves & {$[40,41]$} \\
\hline 164 & $p$-Cymene & Seeds & [7] \\
\hline 165 & Pentacosane & Leaves & [41] \\
\hline 166 & Perilla ketone & Leaves & {$[29,40,44-48]$} \\
\hline 167 & Perillaldehyde & Leaves & {$[40,41,43,45]$} \\
\hline 168 & Perillene & Leaves & {$[41,44]$} \\
\hline 169 & Perillic acid & Leaves & {$[49]$} \\
\hline 170 & Perillyl alcohol & Leaves & {$[40,45]$} \\
\hline
\end{tabular}


Table 2. Cont.

\begin{tabular}{|c|c|c|c|}
\hline Number & Compounds & Parts & References \\
\hline 171 & Piperitenone & Leaves & [41] \\
\hline 172 & $p$-Menth-1-en-4-ol & Leaves & [40] \\
\hline 173 & $p$-Menth-1-en-8-ol & Leaves & [40] \\
\hline 174 & $p$-mentha-2,8-dione & Leaves & [43] \\
\hline 175 & $p$-Mentha-3,8-diene & Leaves & [41] \\
\hline 176 & Pseudolimonene & Leaves & [41] \\
\hline 177 & Pulegone & Leaves & [41] \\
\hline 178 & Phthalic acid & Stems & [7] \\
\hline 179 & Phytol & Leaves & [7] \\
\hline 180 & Phytone & Leaves & [41] \\
\hline 181 & Sabinene & Leaves & {$[40,41,43]$} \\
\hline 182 & Santolina triene & Leaves & [41] \\
\hline 183 & Spathulenol & Leaves & {$[7,40,44]$} \\
\hline 184 & Styrene & Leaves & [41] \\
\hline 185 & Terpinen-4-ol & Leaves & [41] \\
\hline 186 & Terpinolene & Leaves & {$[40,41,43,45]$} \\
\hline 187 & Thujyl alcohol & Leaves & [41] \\
\hline 188 & trans-Furanoid & Leaves & [45] \\
\hline 189 & trans-Nerolidol & Leaves & {$[7,40,41,46]$} \\
\hline 190 & trans-Shisool & Leaves & [40] \\
\hline 191 & Triacontane & Leaves & [40] \\
\hline 192 & Tridecane & Leaves & [41] \\
\hline 193 & Valencene & Leaves & [41] \\
\hline 194 & Valeric acid, pent-2-en-4-ynyl ester & Leaves & [40] \\
\hline 195 & Viridiflorene & Leaves & [41] \\
\hline 196 & Viridiflorol & Leaves & [41] \\
\hline 197 & $\alpha$-Bergamotene & Seeds & {$[7]$} \\
\hline 198 & $\alpha$-Bulnesene & Leaves & [41] \\
\hline 199 & $\alpha$-Cadinol & Leaves & {$[40,41]$} \\
\hline 200 & $\alpha$-Caryophyllene & Leaves & {$[40,43]$} \\
\hline 201 & $\alpha$-Caryophyllene & Leaves & [41] \\
\hline 202 & $\alpha$-Citral & Leaves & {$[40,41]$} \\
\hline 203 & $\alpha$-Copaene & Leaves, Seeds & {$[7,40,41,44]$} \\
\hline 204 & $\alpha$-Curcumene & Stems & [7] \\
\hline 205 & $\alpha$-Farnesene & Leaves, Seeds & {$[7,29,41,43]$} \\
\hline 206 & $\alpha$-Fenchene & Leaves & [41] \\
\hline 207 & $\alpha$-Patchoulene & Leaves & [41] \\
\hline 208 & $\alpha$-Pinene & Leaves & {$[40,41,43]$} \\
\hline 209 & $\alpha$-Santalol & Leaves & [41] \\
\hline 210 & $\alpha$-Terpineol & Stems & [7] \\
\hline 211 & $\alpha$-Terpineol & Leaves & [41] \\
\hline 212 & $\beta$-Cubebene & Leaves & [43] \\
\hline 213 & $\beta$-Bourbonene & Leaves & {$[40,41]$} \\
\hline 214 & $\beta$-Cadinene & Leaves & [41] \\
\hline 215 & $\beta$-Caryophyllene & Stems, Leaves, Seeds & {$[7,29,41,43-46]$} \\
\hline 216 & $\beta$-Citronellene & Leaves & [41] \\
\hline 217 & $\beta$-Cyclocitral & Leaves & [45] \\
\hline 218 & $\beta$-Dehydroelsholtziaketone & Leaves & [41] \\
\hline 219 & $\beta$-Elemene & Leaves & {$[7,40,41]$} \\
\hline 220 & $\beta$-Farnesene & Stems, Leaves, Seeds & {$[7,29,41]$} \\
\hline 221 & $\beta$-Guaiene & Leaves & [40] \\
\hline 222 & $\beta$-Gurjunene & Leaves & [41] \\
\hline 223 & $\beta$-Ionone & Leaves & [41] \\
\hline 224 & $\beta$-Linalool & Leaves & {$[7,29,40,41,44,46]$} \\
\hline 225 & $\beta$-Murolene & Leaves & [45] \\
\hline 226 & $\beta$-Pinene & Leaves & {$[40,41,43]$} \\
\hline 227 & $\beta$-Phellandrene & Leaves & {$[40,41]$} \\
\hline 228 & $\beta$-Selinene & Leaves & [41] \\
\hline 229 & $\beta$-Terpinene & Leaves & [41] \\
\hline 230 & $\gamma$-Pyronene & Leaves & [41] \\
\hline 231 & $\delta$-Cadinene & Leaves & {$[41]$} \\
\hline 232 & $\delta$-Elemene & Leaves & {$[40,41]$} \\
\hline
\end{tabular}

\subsection{Other Terpenoids}

Carotenoids are organic pigments, which belong to the category of tetraterpenoids and are widely distributed in nature, accumulating in chloroplasts. Perilla showed higher carotenoid content, 
even compared to $\beta$-carotene-rich (carrots, spinach) or lutein-rich (spinach, broccoli, lettuce) crops, where the content of carotenoids in perilla is up to five-fold higher [48]. Moreover, trace triterpenes (Table 3, Figure 3), including tormentic acid, oleanolic acid, and ursolic acid, were determined in perilla using high-performance liquid chromatography (HPLC) analysis [50]. There are also some phytosterol (Table 3, Figure 4) compounds (ampesterol, stigmasterol, $\beta$-sitosterol, $\beta$-amyrin, oxalic acid, triacylglycerols) that have been found in perilla seeds. The content of $\beta$-sitosterol was demonstrated to definitely correlate to the content of linolenic acid [51].

\subsection{Fatty Acids and Other Lipid Type Compounds}

Perilla oil constitutes roughly $40 \%$ of the seed weight, and seeds of perilla are a good source of fatty acid composition such as palmitic acid (C17:0), stearic acid (C18:0), oleic acid (C18:1), linoleic acid (C18:2), and linolenic acid (C18:3). In addition, the content of unsaturated fatty acids in perilla seed oils is typically over $90 \%$, contains considerably high levels of $\alpha$-linolenic acid ( $\omega-3$ fatty acid) ( $\alpha$-LNA) ranges from $52.58 \%$ to $61.98 \%$. Furthermore, the $\omega-6$ (linoleic acid) around $14 \%$ and $\omega-9$ (oleic acid) is also present in perilla oil. These polyunsaturated fatty acids are expected to possess various health benefits for humans such as reducing the cholesterol and triglyceride levels in serum, lowering the risk of colon cancer, and preventing the excessive growth of visceral adipose tissue $[3,52,53]$. There are also four types of tocopherols $\left(\alpha-, \beta-, \gamma^{-}\right.$, and $\delta$-tocopherol) that have been found in PF seeds. The content of $\gamma$-tocopherol was demonstrated to definitely correlate to the content of linolenic acid [51] (Table 3, Figure 4).

Table 3. Triterpenes, phytosterols, fatty acids, polycosanol, and tocopherol compounds found in Perilla frutescens species.

\begin{tabular}{|c|c|c|c|}
\hline Number & Compounds & Parts & References \\
\hline \multicolumn{4}{|c|}{ Triterpenes } \\
\hline 233 & 3-Epicorosolic acid & Leaves & [54] \\
\hline 234 & 3-Epimaslinic acid & Leaves & {$[54]$} \\
\hline 235 & Augustic acid & Leaves & {$[54]$} \\
\hline 236 & Corosolic acid & Leaves & [54] \\
\hline 237 & Hyptadienic acid & Leaves & [54] \\
\hline 238 & Oleanolic acid & Leaves & {$[50,54]$} \\
\hline 239 & Pomolic acid & Leaves & [54] \\
\hline 240 & Tormentic acid & Leaves & {$[50,54]$} \\
\hline 241 & Ursolic acid & Leaves & {$[50,54]$} \\
\hline \multicolumn{4}{|c|}{ Phytosterols } \\
\hline 242 & Campesterol & Seeds & [51] \\
\hline 243 & Oxalic acid & Leaves & [55] \\
\hline 244 & Stigmasterol & Seeds & [51] \\
\hline 245 & Triacylglycerols & Seeds & [56] \\
\hline 246 & $\beta$-Amyrin & Seeds & [51] \\
\hline 247 & $\beta$-Sitosterol & Seeds & [51] \\
\hline \multicolumn{4}{|c|}{ Fatty acids } \\
\hline 248 & $5 \alpha$-Cholestane & Seeds & {$[51,53]$} \\
\hline 249 & Arachidic acid & Seeds & [51] \\
\hline 250 & Eicosenoic acid & Seeds & [51] \\
\hline 251 & Lauric acid & Seeds & {$[47]$} \\
\hline 252 & Linoleic acid & Seeds & {$[3,47,51,53,57,58]$} \\
\hline 253 & Linolenic acid & Seeds, Leaves & {$[3,47,51,53,57,58]$} \\
\hline 254 & Oleic acid & Seeds & {$[3,40,47,51,57,58]$} \\
\hline 255 & Palmitic acid & Seeds, Leaves & {$[3,40,47,51,53,57,58]$} \\
\hline 256 & Pentadecanoic acid & Seeds & {$[51]$} \\
\hline 257 & Stearic acid & Seeds & {$[3,47,51,53,57,58]$} \\
\hline 258 & $\beta$-Cholestanol & Seeds & {$[51]$} \\
\hline
\end{tabular}


Table 3. Cont.

\begin{tabular}{cccc}
\hline Number & Compounds & Parts & References \\
\hline & & Polycosanol & \\
\hline 259 & Docosanol & Seeds & {$[51]$} \\
260 & Eicosanol & Seeds & {$[51,56,59]$} \\
261 & Heneicosanol & Seeds & {$[51]$} \\
263 & Heptacosanol & Seeds & {$[51]$} \\
264 & Hexacosanol & Seeds & {$[51]$} \\
265 & Octacosanol & Seeds & {$[51]$} \\
266 & Tetracosanol & Seeds & {$[51]$} \\
267 & Triacontanol & Seeds & {$[51]$} \\
& Tricosanol & Seeds & {$[51]$} \\
\hline 268 & & Tocopherols & {$[51]$} \\
269 & $\alpha$-Tocopherol & Seeds & {$[51]$} \\
270 & $\beta$-Tocopherol & Seeds & {$[51]$} \\
271 & $\gamma$-Tocopherol & Seeds & {$[51]$} \\
\hline
\end{tabular}

\subsection{Policosanols}

Policosanols are very long chain aliphatic alcohols derived from the wax constituent of plants. Contents and compositions of the waxy materials and policosanols (Table 3) were identified and quantified using thin layer chromatography (TLC), HPLC, and GC. Waxy materials, moisture, and crude lipids from perilla seeds comprised about $72 \mathrm{mg} / 100 \mathrm{~g}$, 5.6-8.2\%, and 51.2-48.4\%, respectively, and major components of waxy materials were policosanols (25.5-34.8\%), wax esters, steryl esters, and aldehydes (53.0-49.8\%), hydrocarbons (18.8-10.5\%), acids (1.7-2.1\%), and triacylglycerols (1.0-2.9\%), analyzed using HPLC. Policosanols extracted in the waxy materials of the PF seeds were also determined based on gas chromatography to be $67-68 \%$ octacosanol, $16-17 \%$ hexacosanol, and $6-9 \%$ triacontanol of the total policosanols composition [56]. The seed of perilla was found to be rich in policosanols with $427.83 \mathrm{mg} / \mathrm{kg}$ of oil [59].

\subsection{Nutrients}

The good nutritional value, such as ash content $2.2 \%$, crude fiber $23.28 \%$, crude protein $5.12 \%$, carbohydrates $18.53 \%$, and minerals like calcium 0.238 , magnesium 0.325 , potassium 0.5004 , and phosphorus $0.2124(\mathrm{mg} / \mathrm{g})$, and the fatty acid composition of perilla seed, was also reported [30]. The quality of protein from perilla seed was investigated and an excellent amino acid profile of perilla seed protein was found [60].

\section{Pharmacological Properties of Perilla}

As mentioned earlier, the biological activity of perilla is due to the presence of various biochemical compounds that are responsible for producing health benefits for humans. Because of this, many researchers have focused on the isolation and identification of these active ingredients as well as their biological evaluations.

\subsection{Antioxidant Activity}

Epidemiological, clinical, and nutritional studies show that consumption of so-called functional foods and nutraceuticals may be associated with a lowered risk of cancers, cardiovascular diseases, and metabolic disorders [61]. These benefits are often attributed to the high antioxidant capacity of the drug, and especially to the content of phenolic acids, flavonoids, and carotenoids. It has been reported that extracts from perilla seeds and leaves exhibit concentration-dependent antioxidant activity, based on the 2,2-diphenyl-1-picryl-hydrazyl-hydrate (DPPH) radical assay, and 2,2'-azino-bis(3-ethylbenzothiazoline-6 sulphonic acid) (ABTS) radical cation assay [1]. Isolated rosmarinic acid (RA) and luteolin from the fruit of $P$. frutescens var. acuta showed significant DPPH 
scavenging capacity with half-maximal inhibitory concentration $\left(\mathrm{IC}_{50}\right)$ values of 8.61 and $7.50 \mu \mathrm{M}$, respectively [32]. Similarly, among five phenolic compounds, RA and rosmarinic acid-3-O-glucoside were the dominant phenolic antioxidants with strong activity from cold-pressed perilla var. arguta seed flour studied by Zhou et al. [1]. RA isolated by these authors from perilla leaf exhibited DPPH radical scavenging activity of $88.3 \pm 0.7 \%$ at a concentration of $10 \mu \mathrm{g} / \mathrm{mL}$ with a drug concentration eliciting $50 \%$ of the maximum stimulation (SC50) value of $5.5 \pm 0.2 \mu \mathrm{g} / \mathrm{mL}$. Tian et al. [41] proved that the antioxidant activity of perilla essential oil may depend on the location of cultivation. Extracts of drugs harvested from different regions exhibited varying degrees of scavenging ability at $10 \mathrm{mg} / \mathrm{mL}$ concentrations with an inhibition percentage of $94.80 \pm 0.03 \%$. The $80 \%$ methanol extract of perilla seeds exhibited a strong antioxidant property [62]. In vivo, the protective activity of RA from P. frutescens leaf (PFL) was demonstrated on Lipopolysaccharide (LPS)-induced liver injury of $\mathrm{d}$-GalN-sensitized mice owing to the scavenging or reducing activities of superoxide or peroxynitrite rather than to inhibition of tumor necrosis factor (TNF)- $\alpha$ production [63].

The roles of the flavonoid luteolin from the perilla seeds seems to provide significant antioxidant activity for drugs and extracts. This compound significantly reversed hydrogen peroxide-induced cytotoxicity in primary cultured cortical neurons. Whereas, luteolin markedly attenuated the reactive oxygen species (ROS) production, and prevented the decrease in activities of mitochondria, catalase, and glutathione in ROS-insulted primary neurons, for preventing neurodegenerative diseases [64]. In another study, luteolin inhibited the peroxidation of linoleic acid catalyzed using soybean lipoxygenase- 1 with an $\mathrm{IC}_{50}$ of $5.0 \mathrm{M}(1.43 \mu \mathrm{g} / \mathrm{mL})$ noncompetitively [65].

The monoterpene perillaldehyde was shown to be a potent thioredoxin inducer as it activates the Nrf2-Keap1 system [66]. It seems that the antioxidant activity of perilla may vary among different accessions. As part of an in vitro study in a human subjects, purple perilla leaves showed a higher antioxidant activity, and prevented the oxidation of low-density lipoprotein (LDL) than the green leaves [67]. Another study revealed that $2^{\prime}, 3^{\prime}$-dihydroxy-4' $4^{\prime} 6^{\prime}$-dimethoxychalcone (DDC) found in green perilla leaves enhanced cellular resistance to oxidative damage through activation of the Nrf2-antioxidant response element (ARE) pathway [68].

\subsection{Antibacterial and Antifungal Activity}

Recently, the demand for natural compounds from plant extracts as effective antibacterial agents against a wide range of bacteria is definitely growing to control human infection and for the preservation of food [69]. Perilla seed extract rich in polyphenols was examined for its antibacterial activity against oral cariogenic Streptococci and periodontopathic Porphyromonas gingivalis. The ethyl acetate extracts exhibit strong antibacterial activity against oral Streptococci and various strains of $P$. gingivalis. On the other hand, the ethanolic extract of defatted perilla seed weakly inhibited the growth of oral pathogenic bacterial strains. Among the polyphenols, luteolin showed marked antibacterial activity against the oral bacteria tested [70]. Additionally, the antibacterial activity of the essential oil from perilla leaves on Gram-positive and Gram-negative bacteria was studied, and the results showed the effectiveness of this essential oil to inhibit the growth of the tested bacteria. The minimum inhibitory concentration (MIC) on Staphylococcus aureus and Escherichia coli were $500 \mu \mathrm{g} / \mathrm{mL}$ and $1250 \mu \mathrm{g} / \mathrm{mL}$. respectively [71]. The most abundant terpene-type compound, perillaldehyde, moderately inhibits a broad range of both bacteria in the range of $125-1000 \mathrm{pg} / \mathrm{mL}$. This compound was also particularly active against filament fungi, with MIC values for M. mucedo and P. chrysogenum already at a $62.5 \mathrm{pg} / \mathrm{mL}$ concentration [72]. Kim and Choi [69,73] determined the antibacterial activity of the leaf ethanol extracts of PF var. acuta against $S$. aureus and Pseudomonas aeruginosa and detected that the population of P. aeruginosa decreased from 6.660 to $4.060 \log \mathrm{CFU} / \mathrm{mL}$, and that of S. aureus from 7.535 to $4.865 \log \mathrm{CFU} / \mathrm{mL}$, as well as to $2.600 \log \mathrm{CFU} / \mathrm{mL}$ via extraction with ethyl acetate.

The fungicidal effects of perilla EO were described against Trichophyton mentagrophytes [74], and they dose-dependently decreased the production of $\alpha$-toxin, enterotoxins $\mathrm{A}$ and $\mathrm{B}$, and toxic shock 
syndrome toxin 1 (TSST-1) in both methicillin-sensitive S. aureus and methicillin-resistant S. aureus [75]. The antifungal activity of perilla EO distilled from aerial parts of the plant was also tested against phytopathogenic fungi and its activity was demonstrated in the cases of Aspergillus flavus, Aspergillus oryzae, Aspergillus niger, Rhizopus oryzae, and Alternaria alternate [41].

\subsection{Anti-Allergic Effect}

Studies show that water extracts of PF may inhibit allergic reactions in vivo and in vitro. PF extracts $(0.05$ to $1 \mathrm{~g} / \mathrm{kg})$ greatly inhibited systemic allergic reactions activated by anti-DNP IgE in rats in a dose-dependent manner [76]. Similarly, the water extract of PFL has been shown to have a positive result against atopic dermatitis in an animal model. The anti-allergic effects of PFL on 2,4-dinitrofluorobenzene (DNFB)-induced atopic dermatitis in C57BL/6 mice was evaluated by Heo et al. [77] and the results revealed that an aqueous extract $(100 \mu \mathrm{g} / \mathrm{mL})$ of PFL could significantly inhibit DNFB-induced atopic inflammation by alleviating the expression of MMP-9 and IL-31, as well as augmenting T-bet activity. In another experiment, water extract from PFL significantly suppressed the PCA-reaction, using mice ear-passive cutaneous anaphylaxis (PCA)-reaction, and the authors concluded the role of rosmarinic acid [9]. Application of an ethanol extract from PF, rather than the aqueous extract, suppressed the allergen-specific Th2 responses. Furthermore, airway inflammation and hyperreactivity in an ovoalbumin-sensitized murine model of asthma were alleviated. Based on this, Chen et al. [78] suggested perilla as a potential phytotherapeutic tool for immunomodulation.

Besides using crude extracts, individual compounds as a potential biologically active agent against allergies have also been studied. A novel glycoprotein fraction from the hot water extract of perilla was used and it was found that it moderately inhibited mast cell degranulation and the activities of hyaluronidase $\left(\mathrm{IC}_{50}=0.42 \mathrm{mg} / \mathrm{mL}\right)$ in a dose-dependent manner [79]. Furthermore, daily oral supplementation with RA $(1.5 \mathrm{mg} /$ mouse, orally) from perilla significantly prevented the increase in the numbers of eosinophils in bronchoalveolar lavage fluids and in those around murine airways. Likewise, the expression of IL-4 and IL-5, and eotaxin in the lungs of sensitized mice, together with allergen-specific IgG1, were also inhibited. Due to these findings, the authors revealed RA as an effective intervention against allergic asthma [80]. In other study, perilla extracts enriched with RA could inhibit seasonal allergic rhinoconjunctivitis in humans at least partly via inhibition of polymorphonuclear leukocytes (PMNL) infiltration into the nostrils [81]. The use of a diet supplemented with perilla oil might be effective on asthmatic allergy via decreasing serum lipids and ovalbumin-specific IgG1 and IgA levels in mice [82].

\subsection{Anti-Depressant Activity}

Numerous studies focusing on the extracts and/or purified compounds of $P$. frutescens displayed antidepressant-like effects. Phenolic-type constituents of perilla leaf, such as apigenin, at intraperitoneal doses of 12.5 and $25 \mathrm{mg} / \mathrm{kg}$ [83], RA (2 mg/kg, i.p.) and caffeic acid (4 mg/kg, i.p.) each led to a considerable reduction of the duration of immobility in the forced swimming test. These compounds are also supposed to inhibit the emotional abnormality produced by stress [84,85], which is possibly mediated by the dopaminergic mechanisms in the mouse brain [83].

Essential oils and perillaldehyde from perilla leaves were also found to show an anti-depressant property in mice with CUMS-induced depression [86,87]. In another study, daily consumption of perillaldehyde $(20 \mathrm{mg} / \mathrm{kg}$, oral) demonstrated significant antidepressant-like effects in mice with LPS-induced depression and the authors concluded a potential benefit in inflammation-related depression [88]. Inhaling the same compound (perillaldehyde 0.0965 and $0.965 \mathrm{mg} / \mathrm{mouse} / \mathrm{day}$, 9 days) had antidepressant-like properties on a stress-induced, depression-like model in mice during the forced swim test (FST) through the olfactory nervous function [89].

The oil of PF seeds might have an anti-depressant activity too since a seed oil-rich diet during a forced swim test in adult male rats modulated the fatty acid profiles and brain-derived neurotrophic factor (BDNF) expression in the brain [90]. Moreover, perilla seed oil rich in $n-3$ fatty acids improved 
cognitive function in rats by generating new hippocampal neural membrane structures as well as by inducing specific protein expression [91].

\subsection{Anti-Inflammatory Activity}

Luteolin has been isolated from PFL ethanol extracts and was demonstrated to exert beneficial effects on neuro-inflammatory diseases in a dose-dependent manner $\left(\mathrm{IC}_{50}=6.9 \mu \mathrm{M}\right)$ through suppressing the expression of inducible nitric oxide synthase (iNOS) in BV-2 microglial cells [43].

The ethanol extract of PFL was identified to display significant anti-inflammatory activity in LPS-induced Raw 264.7 mouse macrophages through the inhibition of the expression of pro-inflammatory cytokines, inhibition of mitogen-activated protein kinase (MAPK) activation, and of nuclear factor-kappa (NF-KB) nuclear translocation in response to LPS [92]. The seed oil from PF showed a great protective effect against reflux esophagitis and this could be attributed to the antisecretory (anticholinergic, antihistaminic), antioxidant, and lipoxygenase inhibitory activities due to the presence of $\alpha$-Linolenic acid (ALA) (18:3, n-3) [93]. Furthermore, RA isolated from PFL could inhibit the release of high mobility group box 1 protein (HMGB1) and down-regulated HMGB1-dependent inflammatory responses in human endothelial cells, HMGB1-mediated hyperpermeability, and leukocyte migration in mice, as well as reduced cecal ligation and puncture (CLP)-induced HMGB1 release and sepsis-related mortality. This could be a potential remediation for various vascular inflammatory diseases, such as sepsis and septic shock, via inhibition of the HMGB1 signaling pathway [94]. Lipophilic triterpene acids from ethanol extracts of red and green PFL were demonstrated to have remarkable anti-inflammatory activity on 12-O-tetradecanoylphorbol-13-acetate (TPA)-induced inflammation in mice ( $\mathrm{ID}_{50}: 0.09-0.3 \mathrm{mg}$ per ear), and on the Epstein-Barr virus early antigen (EBV-EA) activation (91-93\% inhibition at $1 \times 10^{3}$ mol ratio/TPA), [54]. A recent study in mice showed that PF extract ameliorated inflammatory bowel disease (IBD) via protection of dextran sulfate sodium-induced murine colitis, with NF- $\mathrm{kB}$ and signal transduction and activator of transcription 3 (STAT3) as putative targets [95]. A perillaketone-type and alkaloid isolated from aerial parts of perilla showed the remarkable inhibitory effect on pro-inflammatory cytokines (TNF- $\alpha$ and/or IL-6) and inflammatory mediator (NO) in LPS-stimulated RAW264.7 cells, indicating that these compounds might be active components for inflammatory disorders [96].

\subsection{Antitumor Effect}

A number of in vivo and in vitro studies have reported the potential anticancer activity of PF. Tormentic acid, a lipophilic triterpene acid from ethanol extracts of red and green PFL, remarkably blocked carcinogenenesis in an in vivo, two-stage mouse skin model [54]. Similarly, in an in vivo carcinogenesis model, topical application of perilla-derived fraction $(2.0 \mathrm{mg} / \mathrm{mouse})$ led to a significant reduction of 7,12-dimethylbenz(a)anthracene (DMBA)-initiated and TPA-promoted tumorgenesis. This is probably based on two independent effects: inhibition of oxidative DNA injury and inhibition of adhesion molecule, chemokine, and eicosanoid synthesis [97].

In addition, Lin et al. [98] evaluated the inhibitory effects of PFL and they found that it effectively induces apoptosis-related genes and could inhibit cell proliferation in human hepatoma HepG2 cells. They also observed that the inhibitory effect of PFL was much higher than the same dose of commercially available RA and luteolin compounds.

In another study, the application of ethanol extract of PFL resulted in induced apoptosis through the combinations of death receptor-mediated, mitochondrial, and endoplasmic reticulum stress-induced pathways, and substantially suppressed the cell proliferation via p21-mediated G1 phase arrest in human leukemia HL-60 cells [99]. Isoegomaketone (IK), an essential oil component of $\mathrm{PF}$, was found to be another potential agent possessing anti-cancer activity. IK induces apoptosis through caspase-dependent and caspase-independent pathways in human colon adenocarcinoma DLD-1 cells [100]. 


\subsection{Miscellaneous Effects}

In addition to the pharmacological activities described above, different extracts, seed oil, and some individual phenolic compounds of perilla have been found to exhibit other special physiological activities indicating further therapeutic utilizations.

An aqueous extract of PF showed potent anti-HIV-1 activity via inhibition giant cell formation in co-culture of Molt- 4 cells with and without HIV- 1 infection showing inhibitory activity against HIV-1 reverse transcriptase [101]. A very recent study indicates the importance of PFL extracts as a potential anti-aging agent for skin, as it showed effectiveness against UV-induced dermal matrix damage in vitro and in vivo [102]. The in vitro neuroprotection activity of unsaturated fatty acids of PF seed oil have been reported by Eckert et al. [103]. Perilla seed oil might be useful for other complaints too. Deng et al. [104] described in vitro and in vivo anti-asthmatic effects of perilla seed oil in the guinea pig and concluded that the oil may ameliorate lung function in asthma by regulating eicosanoid production and suppressing leukotriene (LT) generation. Zhao et al. [105] supposed a possible anti-ischemic activity of luteolin extracted from PFL, likely through a rebalancing of pro-oxidant/antioxidant status.

In vivo, the protective activity of RA from PFL was demonstrated on LPS-induced liver injury of d-GalN-sensitized mice. The treatments significantly reduced the elevation of plasma aspartate aminotransferase (AST) and alanine transaminase (ALT) levels, as well as anti-TNF and sphincter of Oddi dysfunction (SOD) treatment, compared with controls [63]. In one investigation, the hepatoprotective effects of sucrose-treated perilla leaves, other than untreated leaves, exhibited the best result in vitro and in vivo [10].

\section{Toxicology}

Although a well-established application of products from any herb including perilla require proofs not only for efficacy but also for safety. Suprisingly, there are only very few studies that have been reported about the toxicological aspects of materials originating from perilla. Inhaling smoke from roasting perilla seeds led to occupational asthma through an IgE-mediated mechanism [106]. Additionally, a single case of anaphylaxis caused by perilla seed was also reported [107].

\section{Conclusions and Future Perspectives}

P. frutescens L. varieties have a long traditional usage in many Asian countries and now across the world. The plant has been cultivated for multiple usages, traditionally for curing depression-related disease, asthma, anxiety, tumors, coughs, allergies, intoxication, cold, fever, chills, headache, stuffy nose, and some intestinal disorders, and acts as an antioxidant. Due to genetic variations, it has been exploited as an ornamental plant in gardens. Taxonomical aspects of perilla species must be recognized to avoid misleading identification of the plant species via a proper molecular study. The traditional and local uses of the plant are not well documented in the English literature since the plant originally belongs to the Asian countries, and might be the main reason why the ethnobotanical uses of perilla species are not widespread. In addition, it was also used as an edible aromatic vegetable plant to flavor foods. The leaves and seeds have high nutritional value since the leaf is rich in carotenoids and the seed is rich in fatty acid oils, and both have a potential use as functional dietary supplements in food industries. There are 271 active compounds have been reported including phenolic acids, flavonoids, triterpenes, volatile compounds, policosanols, carotenoids, fatty acids, tocopherols, and phytosterols. In addition to the crude solvent extracts, these phytochemicals (rosmarinic acid, perillaldehyde, luteolin, apigenin, tormentic acid, isoegomaketone) are the most studied natural compounds derived from perilla species.

Most of the pharmacological studies outlined in this review are in vitro and in vivo assays that can provide a basis for further studies. The traditional medicinal uses are well-correlated in terms of anti-allergy and anti-depressant activity in which they are claimed for. However, one of the active components of perilla essential oil is perilla ketone, which was found in varied quantities 
in several perilla species and other herbs. This compound has been shown by Kerr et al. [108] to possess pulmonary toxicity in some animals, e.g., horses, sheep, and cows, but there is no evidence for the toxicity of perilla ketone in humans [109]. Toxicological profiles of the active constitutes of perilla, especially aromatic compounds (essential oils), are significantly lacking and need to be addressed. Besides that, the clinical study is not much studied such that further research in this context is necessary to fully ascertain and understand the pharmacological activities and their mechanisms. Although its bioactivity in vitro and in vivo has been revealed to present potential health benefits, such as anti-microbial, antioxidant, anti-allergic, antidepressant, anti-inflammatory, anticancer, and neuroprotective effects, the clinical trials are insufficient to declare a well-established efficacy and safety, therefore human studies are recommended.

Author Contributions: H.M.A. has designed and written the first draft of manuscript. The author has read and approved on the finally submitted version of the manuscript.

Funding: This research received no external funding.

Conflicts of Interest: The author declares that the research was carried out in the absence of any commercial or financial relationships that could be construed as a potential conflict of interest.

$\begin{array}{ll}\text { Abbreviations } & \\ \text { PF } & \text { Perilla frutescens } \\ \text { PFL } & \text { Perilla frutescens leaf } \\ \text { pg/mL } & \text { Picogram/millilitre } \\ \text { UV } & \text { Ultraviolet-visible spectroscopy } \\ \text { NMR } & \text { Nuclear magnetic resonance spectroscopy } \\ \text { ESI-MS } & \text { Electrospray ionisation mass spectrometry } \\ \text { IT-TOF MS } & \text { Ion-trap time-of-flight mass spectrometry } \\ \text { Eos } & \text { Essential oils } \\ \text { SFE-CO2 } & \text { Supercritical fluid extraction } \\ \text { HS-SPME } & \text { Headspace solid phase microextraction } \\ \text { HPLC } & \text { High-performance liquid chromatography } \\ \text { TLC } & \text { Thin layer chromatography } \\ \text { GC-MS } & \text { Gas chromatography-mass spectrometry } \\ \text { ROS } & \text { Reactive oxygen species } \\ \text { DPPH } & \text { 2,2-Diphenyl-1-picryl-hydrazyl-hydrate } \\ \text { ABTS } & \text { 2,2'-Azino-bis(3-ethylbenzothiazoline-6-sulphonic acid) } \\ \text { IC } 50 & \text { Half-maximal inhibitory concentration } \\ \text { RA } & \text { Rosmarinic acid } \\ \text { LPS } & \text { Lipopolysaccharide } \\ \text { d-GalN } & \text { D-galactosamine } \\ \text { TNF-alpha } & \text { Tumor necrosis factor-alpha } \\ \text { LDL } & \text { Low-density lipoprotein } \\ \text { DDC } & \text { 2',3'-Dihydroxy-4',6'-dimethoxychalcone } \\ \text { ARE } & \text { Antioxidant response element } \\ \text { MIC } & \text { Minimum inhibitory concentration } \\ \text { CFU/mL } & \text { Colony-forming units per millilitre } \\ \text { TSST-1 } & \text { Toxic shock syndrome toxin 1 } \\ \text { anti-DNP IgE } & \text { Dinitrophenyl Immunoglobulin } \\ \text { DNFB } & \text { 2,4-Dinitrofluorobenzene } \\ \text { C57BL/6 } & \end{array}$




$\begin{array}{ll}\text { MMP-9 } & \text { Matrix metallopeptidase 9 } \\ \text { IL-31 } & \text { Interleukin 31 } \\ \text { TH2 T } & \text { Helper 2 } \\ \text { PCA } & \text { Passive cutaneous anaphylaxis } \\ \text { PMNL } & \text { Polymorphonuclear leukocytes } \\ \text { FST } & \text { Forced swim test } \\ \text { CUMS } & \text { Chronic unexpected mild stress } \\ \text { BDNF } & \text { Brain-derived neurotrophic factor } \\ \text { Inos } & \text { Inducible nitric oxide synthase } \\ \text { MAPK } & \text { Mitogen-activated protein kinase } \\ \text { NF- } k \text { B } & \text { Nuclear factor-kappa } \\ \text { ALA } & \alpha \text {-Linolenic acid } \\ \text { HMGB1 } & \text { High mobility group box 1 protein } \\ \text { CLP } & \text { Cecal ligation and puncture } \\ \text { TPA } & \text { Tetradecanoylphorbol-13-acetate } \\ \text { EBV-EA } & \text { Epstein-Barr virus early antigen } \\ \text { IBD } & \text { Inflammatory bowel disease } \\ \text { STAT3 } & \text { Signal transducer and activator of transcription 3 } \\ \text { DMBA } & \text { 7,12-Dimethylbenz[a]anthracene } \\ \text { Hep G2 } & \text { Liver hepatocellular cells (a human liver cancer cell line) } \\ \text { IK } & \text { Isoegomaketone } \\ \text { ALT } & \text { Alanine transaminase } \\ \text { AST } & \text { Aspartate aminotransferase } \\ \text { SOD } & \text { Sphincter of oddi dysfunction } \\ & \end{array}$

\section{References}

1. Zhou, X.J.; Yan, L.L.; Yin, P.P.; Shi, L.L.; Zhang, J.H.; Liu, Y.J.; Ma, C. Structural characterisation and antioxidant activity evaluation of phenolic compounds from cold-pressed Perilla frutescens var. arguta seed flour. Food Chem. 2014, 164, 150-157. [CrossRef] [PubMed]

2. Pandey, A.; Bhatt, K.C. Diversity distribution and collection of genetic resources of cultivated and weedy type in Perilla frutescens (L.) Britton var. frutescens and their uses in Indian Himalaya. Genet. Resour. Crop. Evol. 2008, 55, 883-892. [CrossRef]

3. Asif, M. Health effects of omega-3, 6, 9 fatty acids: Perilla frutescens is a good example of plant oils. Orient. Pharm. Exp. Med. 2011, 11, 51-59. [CrossRef] [PubMed]

4. Yu, H.; Qiu, J.F.; Ma, L.J.; Hu, Y.J.; Li, P.; Wan, J.B. Phytochemical and phytopharmacological review of Perilla frutescens L. (Labiatae), a traditional edible-medicinal herb in China. Food Chem. Toxicol. 2017, 108, 375-391. [CrossRef] [PubMed]

5. Chen, Y.P. Application and prescriptions of Perilla in traditional Chinese medicine. In Perilla: The genus Perilla; Kosuna, K., Haga, M., Yu, H.C., Eds.; Harwood Academic Publishers: Amsterdam, The Netherlands, 1997; pp. 37-45.

6. Igarashi, M.; Miyazaki, Y. A review on bioactivities of perilla: Progress in research on the functions of perilla as medicine and food. Evid. Based Complement. Altern. Med. 2013, 2013. [CrossRef] [PubMed]

7. Liu, J.; Wan, Y.; Zhao, Z.; Chen, H. Determination of the content of rosmarinic acid by HPLC and analytical comparison of volatile constituents by GC-MS in different parts of Perilla frutescens (L.) Britt. Chem. Cent. J. 2013, 7. [CrossRef] [PubMed]

8. Ha, T.J.; Lee, J.H.; Lee, M.H.; Lee, B.W.; Kwon, H.S.; Park, C.H.; Shim, K.B.; Kim, H.T.; Baek, I.Y.; Jang, D.S. Isolation and identification of phenolic compounds from the seeds of Perilla frutescens (L.) and their inhibitory activities against $\alpha$-glucosidase and aldose reductase. Food Chem. 2012, 135, 1397-1403. [CrossRef]

9. Makino, T.; Furuta, Y.; Wakushima, H.; Fujii, H.; Saito, K.I.; Kano, Y. Anti-allergic effect of Perilla frutescens and its active constituents. Phytother. Res. 2003, 17, 240-243. [CrossRef]

10. Yang, S.Y.; Hong, C.O.; Lee, H.; Park, S.Y.; Park, B.G.; Lee, K.W. Protective effect of extracts of Perilla frutescens treated with sucrose on tert-butyl hydroperoxide-induced oxidative hepatotoxicity in vitro and in vivo. Food Chem. 2012, 133, 337-343. [CrossRef] 
11. Meng, L.; Lozano, Y.F.; Gaydou, E.M.; Li, B. Antioxidant activities of polyphenols extracted from Perilla frutescens varieties. Molecules 2008, 14, 133-140. [CrossRef]

12. Meng, L.; Lozano, Y.; Bombarda, I.; Gaydou, E.M.; Li, B. Polyphenol extraction from eight Perilla frutescens cultivars. C. R. Chim. 2009, 12, 602-611. [CrossRef]

13. Nitta, M.; Lee, J.K.; Ohnishi, O. Asian Perilla crops and their weedy forms: Their cultivation, utilization and genetic relationships. Econ. Bot. 2003, 57, 245-253. [CrossRef]

14. Brenner, D.M. Perilla: Botany, Uses and Genetic Resources; Wiley: New York, NY, USA, 1993; pp. $322-328$.

15. Sa, K.J.; Choi, S.H.; Ueno, M.; Park, K.C.; Park, Y.J.; Ma, K.H.; Lee, J.K. Identification of genetic variations of cultivated and weedy types of Perilla species in Korea and Japan using morphological and SSR markers. Genes Genom. 2013, 35, 649-659. [CrossRef]

16. Bachheti, R.K.; Joshi, A.; Ahmed, T. A phytopharmacological overview of perilla frutescens. Int. J. Pharm. Sci. Rev. Res. 2014, 26, 55-61.

17. Hu, Y.; Sun, L.W.; Zhang, Y.X.; Wen, C.X.; Xie, X.L.; Liu, Y.J. Primary identifications and palynological observations of Perilla in China. J. Syst. Evol. 2010, 48, 133-145. [CrossRef]

18. Lee, Y.J.; Yang, C.M. Growth behavior and perillaldehyde concentration of primary leaves Kwakof Perilla frutescens (L.) Britton grown in different seasons. Crop. Environ. Biol. 2006, 3, 135-146.

19. Negi, V.S.; Rawat, L.S.; Phondani, P.C.; Chandra, A. Perilla frutescens in transition: A medicinal and oil yielding plant need instant conservation, a case study from Central Himalaya, India. Int. J. Environ. Sci. Technol. 2011, 6, 193-200.

20. Rawat, D.S.; Kharwal, A.D. Ethnobotanical studies of weed flora in Shivalik Hills, Himachal Pradesh, India. Int. J. Adv. Res. 2014, 2, 218-226.

21. Bharali, P.; Sharma, M.; Sharma, C.L.; Singh, B. Ethnobotanical survey of spices and condiments used by some tribes of Arunachal Pradesh. J. Med. Plants Stud. 2017, 5, 101-109.

22. Prakash, A. Uses of some threatened and potential ethnomedicinal plants among the tribals of Uttar Pradesh and Uttrakhand in India. In Proceedings of the National Conference on Forest Biodiversity-Earth's Living Treasure, Lucknow, India, 22 May 2011; pp. 93-99.

23. Dhami, N. Ethnomedicinal uses of plants is Western Terai of Nepal: A case study of Dekhatbhuli VDC of Kanchanpur district. In Medicinal Plants in Nepal: An Anthology of Contemporary Research; Ecological Society: Kathmandu, Nepal, 2008; pp. 165-177.

24. Tiwari, J.K.; Ballabha, R.; Tiwari, P. Some promising wild edible plants of Srinagar and its adjacent area in Alaknanda valley of Garhwal Himalaya, India. J. Am. Sci. 2010, 6, 167-174.

25. Yakang, B.; Gajurel, P.R.; Potsangbam, S.; Bhuyan, L.R. Account of common and traditional non-timber forest products used by Apatani tribe of Arunachal Pradesh, India. Pleione 2013, 7, 514-529.

26. Vlkova, M.; Polesny, Z.; Verner, V.; Banout, J.; Dvorak, M.; Havlik, J.; Krausova, J. Ethnobotanical knowledge and agrobiodiversity in subsistence farming: Case study of home gardens in Phong My commune, central Vietnam. Genet. Resour. Crop. Evol. 2011, 58, 629-644. [CrossRef]

27. Inta, A.; Shengji, P.; Balslev, H.; Wangpakapattanawong, P.; Trisonthi, C. A comparative study on medicinal plants used in Akha's traditional medicine in China and Thailand, cultural coherence or ecological divergence? J. Ethnopharmacol. 2008, 116, 508-517. [CrossRef] [PubMed]

28. Suneetha, J.; Seetharami, R.T.V.V. Ethnomedicine for Rheumatoid Arthritis by the Tribes of East Godavari District, Andhra Pradesh. J. Med. Plant Res. 2016, 6, 1-5.

29. Ito, M.; Honda, G.; Sydara, K. Perilla frutescens var. frutescens in northern Laos. J. Nat. Med. 2008, 62, $251-258$. [CrossRef] [PubMed]

30. Saklani, S.; Chandra, S.; Gautam, A.K. Phytochemical investigation and contribution of Perilla frutescence as spices in traditional health care system. Int. J. Pharm. Technol. 2011, 3, 3543-3554.

31. Peng, Y.; Ye, J.; Kong, J. Determination of phenolic compounds in Perilla frutescens L. by capillary electrophoresis with electrochemical detection. J. Agric. Food Chem. 2005, 53, 8141-8147. [CrossRef]

32. Gu, L.; Wu, T.; Wang, Z. TLC bioautography-guided isolation of antioxidants from fruit of Perilla frutescens var. acuta. LWT Food Sci. Technol. 2009, 42, 131-136. [CrossRef]

33. Gai, F.; Peiretti, P.G.; Karamać, M.; Amarowicz, R. Changes in the Total Polyphenolic Content and Antioxidant Capacities of Perilla (Perilla frutescens L.) Plant Extracts during the Growth Cycle. J. Food Qual. 2017, 2017. [CrossRef] 
34. Yamazaki, M.; Nakajima, J.I.; Yamanashi, M.; Sugiyama, M.; Makita, Y.; Springob, K.; Awazuhara, M.; Saito, K. Metabolomics and differential gene expression in anthocyanin chemo-varietal forms of Perilla frutescens. Phytochemistry 2003, 62, 987-995. [CrossRef]

35. Kang, N.S.; Lee, J.H. Characterisation of phenolic phytochemicals and quality changes related to the harvest times from the leaves of Korean purple perilla (Perilla frutescens). Food Chem. 2011, 124, 556-562. [CrossRef]

36. Raut, J.S.; Karuppayil, S.M. A status review on the medicinal properties of essential oils. Ind. Crop. Prod. 2014, 62, 250-264. [CrossRef]

37. Ito, M.; Toyoda, M.; Kamakura, S.; Honda, G. A new type of essential oil from Perilla frutescens from Thailand. J. Essent. Oil Res. 2002, 14, 416-419. [CrossRef]

38. Zhang, X.; Wu, W.; Zheng, Y.; Chen, L.; Qianrong, C. Essential oil variations in different Perilla L. accessions: Chemotaxonomic implications. Plant Syst. Evol. 2009, 281, 1-10. [CrossRef]

39. Ghimire, B.K.; Yoo, J.H.; Yu, C.Y.; Chung, I.M. GC-MS analysis of volatile compounds of Perilla frutescens Britton var. Japonica accessions: Morphological and seasonal variability. Asian Pac. J. Trop. Med. 2017, 10, 643-651. [CrossRef] [PubMed]

40. Huang, B.; Lei, Y.; Tang, Y.; Zhang, J.; Qin, L.; Liu, J. Comparison of HS-SPME with hydrodistillation and SFE for the analysis of the volatile compounds of Zisu and Baisu, two varietal species of Perilla frutescens of Chinese origin. Food Chem. 2011, 125, 268-275. [CrossRef]

41. Tian, J.; Zeng, X.; Zhang, S.; Wang, Y.; Zhang, P.; Lü, A.; Peng, X. Regional variation in components and antioxidant and antifungal activities of Perilla frutescens essential oils in China. Ind. Crop. Prod. 2014, 59, 69-79. [CrossRef]

42. Ahmed, H.M.; Tavaszi-Sarosi, S. Identification and quantification of essential oil content and composition, total polyphenols and antioxidant capacity of Perilla frutescens (L.) Britt. Food Chem. 2019, 275, 730-738. [CrossRef]

43. Omer, E.A.; Khattab, M.E.; Ibrahim, M.E. First cultivation trial of Perilla frutescens L. in Egypt. Flavour Fragr. J. 1998, 13, 221-225. [CrossRef]

44. Başer, K.H.C.; Demirci, B.; Dönmez, A.A. Composition of the essential oil of Perilla frutescens (L.) Britton from Turkey. Flavour Fragr. J. 2003, 18, 122-123. [CrossRef]

45. Bumblauskiene, L.; Jakstas, V.; Janulis, V.; Mazdzieriene, R.; Ragazinskiene, O. Preliminary analysis on essential oil composition of Perilla L. cultivated in Lithuania. Acta Pol. Pharm. 2009, 66, 409-413. [PubMed]

46. Seo, W.H.; Baek, H.H. Characteristic aroma-active compounds of Korean perilla (Perilla frutescens Britton) leaf. J. Agric. Food Chem. 2009, 57, 11537-11542. [CrossRef] [PubMed]

47. Liu, R.L.; Zhang, J.; Mou, Z.L.; Hao, S.L.; Zhang, Z.Q. Microwave-assisted one-step extraction-derivatization for rapid analysis of fatty acids profile in herbal medicine by gas chromatography-mass spectrometry. Analyst 2012, 137, 5135-5143. [CrossRef]

48. Müller-Waldeck, F.; Sitzmann, J.; Schnitzler, W.H.; Graßmann, J. Determination of toxic perilla ketone, secondary plant metabolites and antioxidative capacity in five Perilla frutescens L. varieties. Food Chem. Toxicol. 2010, 48, 264-270. [CrossRef] [PubMed]

49. Duelund, L.; Amiot, A.; Fillon, A.; Mouritsen, O.G. Influence of the active compounds of Perilla frutescens leaves on lipid membranes. J. Nat. Prod. 2012, 75, 160-166. [CrossRef] [PubMed]

50. Chen, J.H.; Xia, Z.H.; Tan, R.X. High-performance liquid chromatographic analysis of bioactive triterpenes in Perilla frutescens. J. Pharm. Biomed. Anal. 2003, 32, 1175-1179. [CrossRef]

51. Kim, J.K.; Park, S.Y.; Na, J.K.; Seong, E.S.; Yu, C.Y. Metabolite profiling based on lipophilic compounds for quality assessment of perilla (Perilla frutescens) cultivars. J. Agric. Food Chem. 2012, 60, 2257-2263. [CrossRef] [PubMed]

52. Ding, Y.; Hu, Y.; Shi, L.; Chao, M.A.; Liu, Y.J. Characterization of fatty acid composition from five perilla seed oils in China and its relationship to annual growth temperature. J. Med. Plant Res. 2012, 6, 1645-1651.

53. Longvah, T.; Deosthale, Y.G.; Kumar, P.U. Nutritional and short term toxicological evaluation of Perilla seed oil. Food Chem. 2000, 70, 13-16. [CrossRef]

54. Banno, N.; Akihisa, T.; Tokuda, H.; Yasukawa, K.; Higashihara, H.; Ukiya, M.; Nishino, H. Triterpene acids from the leaves of Perilla frutescens and their anti-inflammatory and antitumor-promoting effects. Biosci. Biotechnol. Biochem. 2004, 68, 85-90. [CrossRef]

55. Ogawa, Y.; Takahashi, S.; Kitagawa, R. Oxalate content in common Japanese foods. Acta Urol. J. Hinyokika Kiyo 1984, 30, 305-310. 
56. Adhikari, P.; Hwang, K.T.; Park, J.N.; Kim, C.K. Policosanol content and composition in perilla seeds. J. Agric. Food Chem. 2006, 54, 5359-5362. [CrossRef] [PubMed]

57. Kim, K.S.; Park, S.H.; Choung, M.G. Nondestructive determination of oil content and fatty acid composition in perilla seeds by near-infrared spectroscopy. J. Agric. Food Chem. 2007, 55, 1679-1685. [CrossRef] [PubMed]

58. Schantz, M.M.; Sander, L.C.; Sharpless, K.E.; Wise, S.A.; Yen, J.H.; NguyenPho, A.; Betz, J.M. Development of botanical and fish oil standard reference materials for fatty acids. Anal. Bioanal. Chem. 2013, 405, 4531-4538. [CrossRef] [PubMed]

59. Jung, D.M.; Lee, M.J.; Yoon, S.H.; Jung, M.Y. A Gas Chromatography-Tandem Quadrupole Mass Spectrometric Analysis of Policosanols in Commercial Vegetable Oils. J. Food Sci. 2011, 76, C891-C899. [CrossRef] [PubMed]

60. Longvah, T.; Deosthale, Y.G. Effect of dehulling, cooking and roasting on the protein quality of Perilla frutescens seed. Food Chem. 1998, 63, 519-523. [CrossRef]

61. Zhang, H.; Tsao, R. Dietary polyphenols, oxidative stress and antioxidant and anti-inflammatory effects. Curr. Opin. Food Sci. 2016, 8, 33-42. [CrossRef]

62. Lee, J.H.; Park, K.H.; Lee, M.H.; Kim, H.T.; Seo, W.D.; Kim, J.Y.; Baek, I.Y.; Jang, D.S.; Ha, T.J. Identification, characterisation, and quantification of phenolic compounds in the antioxidant activity-containing fraction from the seeds of Korean perilla (Perilla frutescens) cultivars. Food Chem. 2013, 136, 843-852. [CrossRef] [PubMed]

63. Osakabe, N.; Yasuda, A.; Natsume, M.; Sanbongi, C.; Kato, Y.; Osawa, T.; Yoshikawa, T. Rosmarinic acid, a major polyphenolic component of Perilla frutescens, reduces lipopolysaccharide (LPS)-induced liver injury in D-galactosamine (D-GalN)-sensitized mice. Free Radic. Biol. Med. 2002, 33, 798-806. [CrossRef]

64. Zhao, G.; Yao-Yue, C.; Qin, G.W.; Guo, L.H. Luteolin from Purple Perilla mitigates ROS insult particularly in primary neurons. Neurobiol. Aging 2012, 33, 176-186. [CrossRef]

65. Ha, T.J.; Lee, M.H.; Kim, H.T.; Kwon, H.S.; Baek, I.Y.; Kubo, I.; Jang, D.S.B. Slow-binding inhibition of soybean lipoxygenase-1 by luteolin. Arch. Pharmacal. Res. 2012, 35, 1811-1816. [CrossRef] [PubMed]

66. Masutani, H.; Otsuki, R.; Yamaguchi, Y.; Takenaka, M.; Kanoh, N.; Takatera, K.; Yodoi, J. Fragrant unsaturated aldehydes elicit activation of the Keap1/Nrf2 system leading to the upregulation of thioredoxin expression and protection against oxidative stress. Antioxid. Redox Signal. 2009, 11, 949-962. [CrossRef] [PubMed]

67. Saita, E.; Kishimoto, Y.; Tani, M.; Iizuka, M.; Toyozaki, M.; Sugihara, N.; Kondo, K. Antioxidant activities of Perilla frutescens against low-density lipoprotein oxidation in vitro and in human subjects. J. Oleo Sci. 2012, 61, 113-120. [CrossRef] [PubMed]

68. Izumi, Y.; Matsumura, A.; Wakita, S.; Akagi, K.I.; Fukuda, H.; Kume, T.; Akaike, A. Isolation, identification, and biological evaluation of Nrf2-ARE activator from the leaves of green perilla (Perilla frutescens var. crispa $\mathrm{f}$. viridis). Free Radic. Biol. Med. 2012, 53, 669-679. [CrossRef] [PubMed]

69. Kim, D.H.; Kim, Y.C.; Choi, U.K. Optimization of antibacterial activity of Perilla frutescens var. acuta leaf against Staphylococcus aureus using evolutionary operation factorial design technique. Int. J. Mol. Sci. 2011, 12, 2395-2407. [CrossRef] [PubMed]

70. Yamamoto, H.; Ogawa, T. Antimicrobial activity of perilla seed polyphenols against oral pathogenic bacteria. Biosci. Biotechnol. Biochem. 2002, 66, 921-924. [CrossRef]

71. Qunqun, G. Antibacterial activity of Perilla Frutescens leaf essential oil. Sci. Technol. Food Ind. 2003, 9, 019.

72. Kang, R.; Helms, R.; Stout, M.J.; Jaber, H.; Chen, Z.; Nakatsu, T. Antimicrobial activity of the volatile constituents of Perilla frutescens and its synergistic effects with polygodial. J. Agric. Food Chem. 1992, 40, 2328-2330. [CrossRef]

73. Choi, U.K.; Lee, O.H.; Lim, S.I.; Kim, Y.C. Optimization of antibacterial activity of Perilla frutescens var. acuta leaf against Pseudomonas aeruginosa using the evolutionary operation-factorial design technique. Int. J. Mol. Sci. 2010, 11, 3922-3932. [CrossRef]

74. Inouye, S.; Nishiyama, Y.; Hasumi, Y.; Yamaguchi, H.; Abe, S.; Uchida, K. The vapor activity of oregano, perilla, tea tree, lavender, clove, and geranium oils against a Trichophyton mentagrophytes in a closed box. J. Infect. Chemother. 2006, 12, 349-354. [CrossRef]

75. Qiu, J.; Zhang, X.; Luo, M.; Li, H.; Dong, J.; Wang, J.; Deng, X. Subinhibitory concentrations of perilla oil affect the expression of secreted virulence factor genes in Staphylococcus aureus. PLoS ONE 2011, 6. [CrossRef] 
76. Shin, T.Y.; Kim, S.H.; Kim, S.H.; Kim, Y.K.; Park, H.J.; Chae, B.S.; Kim, H.M. Inhibitory effect of mast cell-mediated immediate-type allergic reactions in rats by Perilla frutescens. Immunopharmacol. Immunotoxicol. 2000, 22, 489-500. [CrossRef] [PubMed]

77. Heo, J.C.; Nam, D.Y.; Seo, M.S.; Lee, S.H. Alleviation of atopic dermatitis-related symptoms by Perilla frutescens Britton. Int. J. Mol. Med. 2011, 28, 733-737. [PubMed]

78. Chen, M.L.; Wu, C.H.; Hung, L.S.; Lin, B.F. Ethanol extract of Perilla frutescens suppresses allergen-specific Th2 responses and alleviates airway inflammation and hyperreactivity in ovalbumin-sensitized murine model of asthma. J. Evid. Based Complement. Altern. Med. 2015, 2015. [CrossRef]

79. Asada, M.; Fukumori, Y.; Inoue, M.; Nakagomi, K.; Sugie, M.; Fujita, Y.; Oka, S. Glycoprotein derived from the hot water extract of mint plant, Perilla frutescens Britton. J. Agric. Food Chem. 1999, 47, 468-472. [CrossRef] [PubMed]

80. Sanbongi, C.; Takano, H.; Osakabe, N.; Sasa, N.; Natsume, M.; Yanagisawa, R.; Yoshikawa, T. Rosmarinic acid in perilla extract inhibits allergic inflammation induced by mite allergen, in a mouse model. Clin. Exp. Allergy 2004, 34, 971-977. [CrossRef] [PubMed]

81. Takano, H.; Osakabe, N.; Sanbongi, C.; Yanagisawa, R.; Inoue, K.I.; Yasuda, A.; Yoshikawa, T. Extract of Perilla frutescens enriched for rosmarinic acid, a polyphenolic phytochemical, inhibits seasonal allergic rhinoconjunctivitis in humans. Exp. Biol. Med. 2004, 229, 247-254. [CrossRef]

82. Chang, H.H.; Chen, C.S.; Lin, J.Y. Dietary perilla oil lowers serum lipids and ovalbumin-specific IgG1, but increases total IgE levels in ovalbumin-challenged mice. Food Chem. Toxicol. 2009, 47, 848-854. [CrossRef]

83. Nakazawa, T.; Yasuda, T.; Ueda, J.; Ohsawa, K. Antidepressant-like effects of apigenin and 2, 4, 5-trimethoxycinnamic acid from Perilla frutescens in the forced swimming test. Biol. Pharm. Bull. 2003, 26, 474-480. [CrossRef]

84. Takeda, H.; Tsuji, M.; Inazu, M.; Egashira, T.; Matsumiya, T. Rosmarinic acid and caffeic acid produce antidepressive-like effect in the forced swimming test in mice. Eur. J. Pharmacol. 2002, 449, 261-267. [CrossRef]

85. Takeda, H.; Tsuji, M.; Miyamoto, J.; Matsumiya, T. Rosmarinic acid and caffeic acid reduce the defensive freezing behavior of mice exposed to conditioned fear stress. Psychopharmacology 2002, 164, 233-235. [CrossRef]

86. Yi, L.T.; Li, J.; Geng, D.; Liu, B.B.; Fu, Y.; Tu, J.Q.; Weng, L.J. Essential oil of Perilla frutescens-induced change in hippocampal expression of brain-derived neurotrophic factor in chronic unpredictable mild stress in mice. J. Ethnopharmacol. 2013, 147, 245-253. [CrossRef]

87. Ji, W.W.; Li, R.P.; Li, M.; Wang, S.Y.; Zhang, X.; Niu, X.X.; Li, W.; Yan, L.; Wang, Y.; Fu, Q.; et al. Antidepressant-like effect of essential oil of Perilla frutescens in a chronic, unpredictable, mild stress-induced depression model mice. Chin. J. Nat. Med. 2014, 12, 753-759. [CrossRef]

88. Ji, W.W.; Wang, S.Y.; Ma, Z.Q.; Li, R.P.; Li, S.S.; Xue, J.S.; Fu, Q. Effects of perillaldehyde on alternations in serum cytokines and depressive-like behavior in mice after lipopolysaccharide administration. Pharmacol. Biochem. Behav. 2014, 116, 1-8. [CrossRef] [PubMed]

89. Ito, N.; Nagai, T.; Oikawa, T.; Yamada, H.; Hanawa, T. Antidepressant-like effect of l-perillaldehyde in stress-induced depression-like model mice through regulation of the olfactory nervous system. J. Evid. Based Complement. Altern. Med. 2011, 2011. [CrossRef] [PubMed]

90. Lee, H.C.; Ko, H.K.; Huang, B.E.G.; Chu, Y.H.; Huang, S.Y. Antidepressant-like effects of Perilla frutescens seed oil during a forced swimming test. Food Funct. 2014, 5, 990-996. [CrossRef] [PubMed]

91. Lee, J.; Park, S.; Lee, J.Y.; Yeo, Y.K.; Kim, J.S.; Lim, J. Improved spatial learning and memory by perilla diet is correlated with immunoreactivities to neurofilament and $\alpha$-synuclein in hilus of dentate gyrus. Proteome Sci. 2012, 10. [CrossRef] [PubMed]

92. Huang, B.P.; Lin, C.H.; Chen, Y.C.; Kao, S.H. Anti-inflammatory effects of Perilla frutescens leaf extract on lipopolysaccharide-stimulated RAW264. 7 cells. Mol. Med. Rep. 2014, 10, 1077-1083. [CrossRef]

93. Arya, E.; Saha, S.; Saraf, S.A.; Kaithwas, G. Effect of Perilla frutescens fixed oil on experimental esophagitis in albino Wistar rats. BioMed Res. Int. 2013, 2013. [CrossRef]

94. Yang, E.J.; Ku, S.K.; Lee, W.; Lee, S.; Lee, T.; Song, K.S.; Bae, J.S. Barrier protective effects of rosmarinic acid on HMGB1-induced inflammatory responses in vitro and in vivo. J. Cell. Physiol. 2013, 228, 975-982. [CrossRef] 
95. Park, D.D.; Yum, H.W.; Zhong, X.; Kim, S.H.; Kim, S.H.; Kim, D.H.; Surh, Y.J. Perilla frutescens extracts protects against dextran sulfate sodium-induced murine colitis: NF-kB, STAT3, and Nrf2 as putative targets. Front. Pharmacol. 2017, 8. [CrossRef]

96. Wang, X.F.; Li, H.; Jiang, K.; Wang, Q.Q.; Zheng, Y.H.; Tang, W.; Tan, C.H. Anti-inflammatory constituents from Perilla frutescens on lipopolysaccharide-stimulated RAW264. 7 cells. Fitoterapia 2018, 130, 61-65. [CrossRef]

97. Osakabe, N.; Yasuda, A.; Natsume, M.; Yoshikawa, T. Rosmarinic acid inhibits epidermal inflammatory responses: Anticarcinogenic effect of Perilla frutescens extract in the murine two-stage skin model. Carcinogenesis 2004, 25, 549-557. [CrossRef] [PubMed]

98. Lin, C.S.; Kuo, C.L.; Wang, J.P.; Cheng, J.S.; Huang, Z.W.; Chen, C.F. Growth inhibitory and apoptosis inducing effect of Perilla frutescens extract on human hepatoma HepG2 cells. J. Ethnopharmacol. 2007, 112, 557-567. [CrossRef]

99. Kwak, C.S.; Yeo, E.J.; Moon, S.C.; Kim, Y.W.; Ahn, H.J.; Park, S.C. Perilla leaf, Perilla frutescens, induces apoptosis and G1 phase arrest in human leukemia HL-60 cells through the combinations of death receptor-mediated, mitochondrial, and endoplasmic reticulum stress-induced pathways. J. Med. Food 2009, 12, 508-517. [CrossRef] [PubMed]

100. Cho, B.O.; Jin, C.H.; Park, Y.D.; Ryu, H.W.; Byun, M.W.; Seo, K.I.; Jeong, I.Y. Isoegomaketone induces apoptosis through caspase-dependent and caspase-independent pathways in human DLD1 cells. Biosci. Biotechnol. Biochem. 2011, 75, 1306-1311. [CrossRef] [PubMed]

101. Yamasaki, K.; Nakano, M.; Kawahata, T.; Mori, H.; Otake, T.; Ueda, N.; Murata, H. Anti-HIV-1 activity of herbs in Labiatae. Biol. Pharm. Bull. 1998, 21, 829-833. [CrossRef]

102. Bae, J.S.; Han, M.; Shin, H.S.; Kim, M.K.; Shin, C.Y.; Lee, D.H.; Chung, J.H. Perilla frutescens leaves extract ameliorates ultraviolet radiation-induced extracellular matrix damage in human dermal fibroblasts and hairless mice skin. J. Ethnopharmacol. 2017, 195, 334-342. [CrossRef]

103. Eckert, G.P.; Franke, C.; Nöldner, M.; Rau, O.; Wurglics, M.; Schubert-Zsilavecz, M.; Müller, W.E. Plant derived omega-3-fatty acids protect mitochondrial function in the brain. Pharmacol. Res. 2010, 61, $234-241$. [CrossRef]

104. Deng, Y.M.; Xie, Q.M.; Zhang, S.J.; Yao, H.Y.; Zhang, H. Anti-asthmatic effects of perilla seed oil in the guinea pig in vitro and in vivo. Planta Med. 2007, 73, 53-58. [CrossRef]

105. Zhao, G.; Zang, S.Y.; Jiang, Z.H.; Chen, Y.Y.; Ji, X.H.; Lu, B.F.; Guo, L.H. Postischemic administration of liposome-encapsulated luteolin prevents against ischemia-reperfusion injury in a rat middle cerebral artery occlusion model. J. Nutr. Biochem. 2011, 22, 929-936. [CrossRef] [PubMed]

106. Jung, S.; Lee, W.Y.; Yong, S.J.; Shin, K.C.; Kim, C.W.; Lee, J.H.; Kim, S.H. Occupational asthma caused by inhaling smoke from roasting perilla seeds. Allergy Asthma Respir. Dis. 2013, 1, 90-93. [CrossRef]

107. Jeong, Y.Y.; Park, H.S.; Choi, J.H.; Kim, S.H.; Min, K.U. Two cases of anaphylaxis caused by perilla seed. J. Allergy Clin. Immunol. 2006, 117, 1505-1506. [CrossRef] [PubMed]

108. Kerr, L.A.; Johnson, B.J.; Burrows, G.E. Intoxication of cattle by Perilla frutescens (purple mint). Vet. Hum. Toxicol. 1986, 28, 412-416. [PubMed]

109. Bassoli, A.; Borgonovo, G.; Morini, G.; De Petrocellis, L.; Moriello, A.S.; Di Marzo, V. Analogues of perillaketone as highly potent agonists of TRPA1 channel. Food Chem. 2013, 141, 2044-2051. [CrossRef]

Sample Availability: Samples of the compounds are not available from the authors. 